\title{
ASYMPTOTIC BEHAVIOR OF FINITE-TIME RUIN PROBABILITY IN A BY-CLAIM RISK MODEL WITH CONSTANT INTEREST RATE
}

\author{
Lei Wang \\ Department of Mathematics, The University of Southern Mississippi, USA
}

Received 2014-05-07; Revised 2014-06-09; Accepted 2014-08-04

\begin{abstract}
This study investigates the ruin probability of a renewal risk model with constant interest rate and by-claim parts. We assume that the claim size and the inter-arrival time satisfy a certain dependent structure with some additional assumptions on their distribution functions. In particular, we study the asymptotic behavior of $P\left(\mathrm{R}_{\delta}^{*}(t, \mathrm{x})>\mathrm{x}\right)$, which holds uniformly in a finite interval. In this way, we significantly extend the Li's result regarding the pairwise strong quasi-asymptotically independent random variables.
\end{abstract}

Keywords: Renewal Risk Model, Subexponential Distribution, Uniform Asymptotic, Pairwise Strong Quasi-Asymptotically

\section{INTRODUCTION}

Risk theory plays an important role in financial mathematics and actuarial science. It has been studied by many domestic and foreign scholars. A variety of risk models with many special features also have been investigated by many papers in the literature. They provide us different perspectives to understand the risk model and related theories. About a century ago, Lundberg (1903) laid the foundation of actuarial risk model in his Uppsala thesis. Waters and Papatriandafylou (1983) introduced delay in claims settlements in a discrete-time risk model and applied martingale techniques to derive upper bounds for ruin probabilities. In addition, Yuen et al. (2005) applied the probability-generating functions to obtain ruin probabilities for the compound binomial model with discrete delay time for by-claim. In addition, Tang (2005) investigated a simple asymptotic formula for the ruin probability of the renewal risk model with constant interest force and regularly varying tailed claims. Recently, Weng et al. (2012) was interested in the tail probability of the Poisson shot noise process and established some asymptotic formulas for the finite and infinite ruin probabilities of a continuous time risk model. Corresponding results can also be found in (Chen and Ng, 2007; Wang, 2008; Li et al., 2009; Yang and Wang, 2010) and some others.

Consider renewal risk model with the total capital reserve up to time $t$, denoted by $R_{\delta}(t, x)$ as expressed by the following equilibrium Equation 1.1:

$$
\begin{aligned}
& R_{\delta}(t, \mathrm{x})=x e^{\delta t} \\
& +\int_{0}^{t} e^{\delta(t-s)} c d s-\sum_{k=1}^{\infty} X_{k} e^{\delta\left(t-\omega_{k}\right)} 1_{\left\{\omega_{k} \leq t\right\}} \\
& -\sum_{k=1}^{\infty} Z_{k} e^{\delta\left(t-\omega_{k}-T_{k}\right)} 1_{\left\{\omega_{k}+T_{k} \leq t\right\}}
\end{aligned}
$$

where, $x$ denotes the initial capital of the insurance company, $\delta>0$ is the constant interest rate and $c$ is the constant gross premium rate, $x e^{\delta t}$ denotes the total capital after time $t$.

We have already known the general formula of the compound-interest:

$$
P=x\left(1+\frac{\delta}{\mathrm{m}}\right)^{m t}
$$


$P$ is the amount of money accumulated after $t$ years, $x$ is the principal amount, $t$ is the number of years the amount is deposited or borrowed for, $m$ is the number of times the interest is compounded per year and $\delta$ is the annual rate of interest. Then we start the compounding more and more, the frequency of compounding include yearly, half-yearly, quarterly, monthly, daily, even if $t$ goes to infinity and then we derive that:

$$
\mathrm{P}=\operatorname{xim}_{m \rightarrow \infty}\left(1+\frac{\delta}{\mathrm{m}}\right)^{m t}=x e^{\delta t}
$$

In this renewal risk model, the deterministic linear function ct denotes the total amount of premiums accumulated up to time $t \geq 0$. Then $\tilde{c}(t)=\int_{0}^{t} e^{\delta(t-s)} c d s=\frac{c}{\delta}\left(e^{\delta t}-1\right) \quad$ denotes total capital generated by the premiums by the time $t \geq 0$.

Consider the risk model in which the claim sizes and the arrival times of successive claims fulfill the following requirements:

- The claims sizes: $X_{k}, k \geq 1$ constitute a sequence of not necessarily independent and nonnegative random variables with common distribution $\mathrm{H}$ :

$$
H(x)=1-H(x)=P\{\geq x\}>0 \text { for all } x>0
$$

- The arrival times of successive claims are: $\omega_{k}=\sum_{k=1}^{n} Y_{k}, k \geq 1$. The inter-arrival time $\left\{Y_{k} ; k \geq 1\right\}$ form a sequence of random variables with common distribution function $V$, but are not necessarily independent. The arrival times of successive claims can generate a renewal counting process Equation 1.2:

$$
N(\mathrm{t})=\sum_{k=1}^{\infty} 1_{\left\{\omega_{k} \leq t\right\}}
$$

where, $1_{A}$ is the indicator function of an event $A$. Then $N(t)$ also describes the total number of claims in finite interval $[0, t]$. Denote the renewal function by $\lambda(t)=$ $\mathrm{E} N(t), t \leq 0$ and assume that $\lambda(t)<\infty$ for all $0<t<\infty$ that:

$$
\Lambda\left\{\begin{array}{l}
{[\underline{t}, \infty] P\left(Y_{1}=\underline{t}\right)>0} \\
(\underline{t}, \infty] P\left(Y_{1}=\underline{t}\right)=0
\end{array}\right.
$$

- We assume that $\left\{X_{k} ; k \geq 1\right\},\left\{Y_{k} ; k \geq 1\right\}$ and $\{c(t) ; t \geq 0\}$ are mutually independent
- In our risk model, there are two parts of mutually independent claims, namely main claims and byclaims Equation 1.3:

$$
\begin{aligned}
& \mathrm{R}_{\delta}^{*}(t, \mathrm{x})=\sum_{k=1}^{\infty} X_{k} e^{\delta\left(t-\omega_{k}\right)} 1_{\left\{\omega_{k} \leq t\right\}} \\
& +\sum_{k=1}^{\infty} Z_{k} e^{\delta\left(t-\omega_{k}-T_{k}\right)} 1_{\left\{\omega_{k}+T_{k} \leq t\right\}}
\end{aligned}
$$

We refer $Z_{n}$ as by-claims or delayed claims in the renewal risk model. They are identically distributed with common distribution $F$. They are usually induced by the main claim with some probability and the occurrence of a by-claim may be delayed depending on associated main claims amount. If the main claim occurs at the $\omega_{k}$, then the by-claim occurs at the $T_{k}+\omega_{k}$. Let $T_{k}, k \geq 1$, be the corresponding delay times of the by-claim and they are identically distributed with common distribution function $\mathrm{G}$ and form a sequence random variables, which are nonnegative, but possibly generated at 0 . In this study, we assume that the $\left\{X_{n}, Z_{n} ; n \geq 1\right\}$ and $\left\{T_{n} ; n \geq 1\right\}$ are mutually independent.

The claims can produce the dependent influence on each other and some additional damages and costs, such as a tornado, hurricane and heavy rain-storm and so on. We define them as by-claim model. Hence, our renewal model with by-claim parts can better reflect the truth.

The ultimate ruin probability in the infinite time is defined as Equation 1.4:

$\Phi(x)=P\left(R_{\delta}(x, \mathrm{~s})<0\right.$, for somes $\left.\geq 0\right)$

And the ruin probability in the finite time interval $[0$, $t$ ] is given by Equation 1.5:

$\Phi(x, t)=P\left(R_{\delta}(x, \mathrm{~s})<0\right.$, for some $\left.0 \leq \mathrm{s} \leq \mathrm{t}\right)$

The ruin probability in the finite time means the insurer's capital falls below zero in the finite time interval $[0, t]$, that is, the total claim exceeds the initial capital plus premium income. We also investigate the asymptotic behavior of the ruin probability in the finite time. Once the capital is less than zero, the ruin occurs and the company will bankrupt.

\section{PRELIMINARIES}

We first introduce some notations. Throughout the paper, all limit relationships are for $x$ tending to infinite unless otherwise stated. Define: 


$$
a(\mathrm{x})=o(b(\mathrm{x})) \text { if } \lim a(\mathrm{x}) / b(\mathrm{x})=0
$$

Furthermore, for two positive bivariate functions $a$ $(\cdot, \cdot)$ and $b(\cdot, \cdot)$ Equation 2.1:

$$
a(\mathrm{x}) \sim b(\mathrm{x}) \text { if } \lim _{x \rightarrow \infty} \sup _{t \in \Delta}\left|\frac{a(\mathrm{x}, \mathrm{t})}{b(\mathrm{x}, \mathrm{t})}-1\right|=0
$$

Clearly, the asymptotic relation $a(x, t) \sim b(x, t)$ holds uniformly for $t \in \Delta$ :

$$
\lim \sup _{x \rightarrow \infty} \sup _{t \in \Delta}\left|\frac{a(\mathrm{x}, \mathrm{t})}{b(\mathrm{x}, \mathrm{t})}-1\right| \leq 1
$$

And:

$$
\lim \inf _{x \rightarrow \infty} \inf _{t \in \Delta}\left|\frac{a(\mathrm{x}, \mathrm{t})}{b(\mathrm{x}, \mathrm{t})}-1\right| \geq 1
$$

As is the case for many recent references in the fields of risk theory, we are interested in ruin probabilities in finite interval under the assumption that $H$ is heavytailed. In particular, the integrated-tail distribution of $H$ is subexponential.

For distribution $H(x)$ on the $(-\infty, \infty), \bar{H}(x)=1-H(x)$ is the tail distribution of the function $H$. We denote the upper and lower Matuszewska index of $H(x)$ :

$$
\begin{aligned}
& J_{H}^{+}-\lim _{x \rightarrow \infty} \frac{\log \bar{H}_{L}(\mathrm{x})}{\log x}, \bar{H}_{L}(\mathrm{x}) \\
& =\lim _{x \rightarrow \infty} \inf \frac{\bar{H}(\mathrm{xy})}{\bar{H}(\mathrm{x})} \text { for } y>1 \\
& J_{H}^{-}-\lim _{x \rightarrow \infty} \frac{\log \bar{H}_{U}(\mathrm{x})}{\log x}, \bar{H}_{U}(\mathrm{x}) \\
& =\lim _{x \rightarrow \infty} \sup \frac{\bar{H}(\mathrm{xy})}{\bar{H}(\mathrm{x})} \text { for } y>1
\end{aligned}
$$

A d.f. $H$ with support $(0, \infty)$ is subexponential, if for all $n \geq 2$ :

$$
\lim _{x \rightarrow \infty} \frac{\overline{H^{* n}}(x)}{n \bar{H}(x)}=1
$$

where, the $\overline{H^{* n}}(x)$ denotes the n-fold convolution of $H$. (Tang, 2006).
The class of subexponential distribution functions will be denoted by $S$. The class of subexponential distribution plays a crucial role in heavy-tailed distribution. In the insurance industry, practitioners usually choose heavy-tailed random variables to model large claims, so we have included for a more detailed analysis of their properties.

A sufficient condition for subexponentiality, if there is an integer $n \geq 2$, such that:

$$
\lim _{x \rightarrow \infty} \frac{\overline{H^{* n}}(x)}{\bar{H}(x)} \leq n
$$

Then $H \in S$ :

Therefore, under the hypothesis of the formula, we obtain:

$$
\lim _{x \rightarrow \infty} \frac{\overline{H^{* 2}}(x)}{\bar{H}(x)} \leq 2
$$

The class of dominated varying distribution is defined as:

$$
D=\left\{H \text { df on }(0, \infty): \lim _{x \rightarrow \infty} \sup \frac{\bar{H}(\mathrm{xy})}{\bar{H}(\mathrm{y})}<\infty, \text { for any } \mathrm{y}>0\right\}
$$

If we suppose $H \in D$, then for any $\eta>J_{H}^{+}$, there exist two positive constants $c$ and $d$ such that when $x \geq y \geq d$ :

$$
\frac{\bar{H}(\mathrm{y})}{\bar{H}(\mathrm{x})} \leq c\left(\frac{\mathrm{x}}{\mathrm{y}}\right)^{\eta}
$$

We have already encountered members of the following three families:

The definition of the class of long-tailed distribution:

$$
\mathcal{L}=\left\{H \mathrm{df} \text { on }(0, \infty): \lim _{x \rightarrow \infty} \sup \frac{\bar{H}(\mathrm{x}+\mathrm{y})}{\bar{H}(\mathrm{y})}=1, \text { for any } \mathrm{y}>0\right\}
$$

We can see that a distribution $H \in \mathcal{L}$ and only if there exists a function $1(\cdot)$ : $[0, \infty) \longmapsto[0, \infty)$ such that $l(x) \rightarrow$ $\infty l(x)=\mathrm{o}(x)$ and:

$$
\bar{H}(x+y) \sim \bar{H}(x)
$$

Holds uniformly for all $|y| \leq l(x)$. 
According to the definition, we may define a little bigger distribution $H \in E R V$ on $[0, \infty)$, if there are some $0<\alpha \leq \beta<\infty$ such that:

$$
s^{-\beta} \leq \liminf \frac{\bar{H}(\mathrm{sx})}{\bar{H}(\mathrm{x})} \leq \limsup \frac{\bar{H}(\mathrm{sx})}{\bar{H}(\mathrm{x})} \leq s^{-\alpha}, \text { forall } \mathrm{s} \geq 1
$$

Which denoted by $H \in E R V(-\alpha,-\beta)$. If $\alpha=\beta$, we say that $H$ belongs to the regular variation class and write $H \in R-\alpha$.

It is well known that the following proper inclusion relationship should hold for the distribution of heavy-tail:

$$
R-{ }_{a} \subset E R V(-\alpha,-\beta) \subset \mathcal{L} \cap \mathcal{D} \subset \mathcal{D}
$$

Embrechts et al. (1997; Kluppelberg and Stadtmuller, 1998; Tang and Tsitsiashvili, 2003; Li et al., 2009; Hao and Tang, 2008) From the study of many contexts and literatures, we easily found that the renewal risk model with constant interest rate mainly involves the independent structure between the claim sizes and arrival times of successive claims; this limits the usefulness of the obtained results to some extent. However, the introduction of dependent structure to risk models has captured more and more researchers' attention in recent years and it provides a special perspective for the ruin probabilities theory. Many previous papers have already worked on this new topic, for example, Yang and Wang (2010; Liu et al., 2012; Wang et al., 2013) and others. The dependent structure also allows the underlying random variables to be positive or negative. Hence, we simply summarize the current corresponding results and make clear the relationship between them. It is also necessary for our proof. We further extend the study to the dependent case and get several similar results about the dependent random variables.

We may define random variables $\left\{\xi_{i}, i \geq 1\right\}$ as Lower Negatively Dependent (LND) and Upper Negatively Dependent (UND) if for each $i \geq 1$ and all $x_{1} \ldots x_{i}$ :

$$
\begin{gathered}
P\left(\bigcap_{i=1}^{n}\left\{\xi_{i} \leq x_{i}\right\}\right) \leq \prod_{i=1}^{n} P\left(\xi_{i} \leq x_{i}\right) \\
P\left(\bigcap_{i=1}^{n}\left\{\xi_{i}>x_{i}\right\}\right) \leq \prod_{i=1}^{n} P\left(\xi_{i}>x_{i}\right)
\end{gathered}
$$

If the sequence can satisfy both the LND and UND, we can name it Negatively Dependent (ND) structure. When $n=2$, the LND, UND and ND structures are equivalent (Lehmann, 1966).
We say that two random variables $\left\{\xi_{i}, i \geq 1\right\}$ are pairwise Negatively Quadrant Dependent (NQD), if for all positive integers $i \neq j, \xi_{i}$ and $\xi_{i}$ are NQD:

$$
P\left(\xi_{1} \leq x_{1}, \xi_{2} \leq x_{2}\right) \leq P\left(\xi_{1} \leq x_{1}\right) P\left(\xi_{2} \leq x_{2}\right)
$$

Or equivalently:

$$
P\left(\xi_{1},>x_{1}, \xi_{2}>x_{2}\right) \leq P\left(\xi_{1}>x_{1}\right) P\left(\xi_{2}>x_{2}\right)
$$

Additionally, we also named the LND as the NLOD in Li et al. (2009) with different notations and different formulas.

We can define that $\left\{\xi_{n}, n \geq 1\right\}$ are WUOD, widely upper orthant dependent. If there exists a finite real sequence $\left\{g_{U(n)} n \geq 1\right\}$ satisfying for each $n \geq 1$ and for all $x_{i} \in(-\infty, \infty), 1 \leq i \leq n$ :

$$
P\left(\bigcap_{i=1}^{n}\left\{\xi_{i}>x_{i}\right\}\right) \leq g_{U}(u) \prod_{i=1}^{n} P\left(\xi_{i}>x_{i}\right)
$$

We can also define that $\left\{\xi_{n}, n \geq 1\right\}$ are WLOD, widely lower orthant dependent. If there exists a finite real sequence $\left\{g_{L(n)}, n \geq 1\right\}$ satisfying for each $n \geq 1$ and for all $x_{i} \in(-\infty, \infty), 1 \leq i \leq n$, :

$$
P\left(\bigcap_{i=1}^{n}\left\{\xi_{i} \leq x_{i}\right\}\right) \leq g_{L}(n) \prod_{i=1}^{n} P\left(\xi_{i}>x_{i}\right)
$$

Accordingly, we would like remark that if the $\left\{\xi_{n}\right.$, $n \geq 1\}$ can hold (2.9) and (2.10), it is also said to be WOD, widely orthant dependent (Wang et al., 2013):

- And then we should be familiar with some important properties of the WUOD and WLOD

- Firstly, we suppose $\left\{\xi_{n}, n \geq 1\right\}$ satisfy the WLOD or WUOD

- If $\left\{f_{n}(\cdot), n \geq 1\right\}$ are non-decreasing, then $\left\{f_{n}\left(\xi_{n}\right), n \geq 1\right\}$ are still WLOD or WUOD

- Inversely, if $\left\{f_{n}(\cdot), n \geq 1\right\}$ are non-increasing, then $\left\{f_{n}\left(\xi_{n}\right), n \geq 1\right\}$ are WUOD or WLOD

- Secondly, if $\left\{\xi_{n}, n \geq 1\right\}$ are nonnegative and WUOD, then for each $n \geq 1$ :

$$
E \prod_{i=1}^{n} \xi_{i} \leq g_{U}(n) \prod_{i=1}^{n} E \xi_{i}
$$

In particular, if the $\left\{\xi_{n}, n \geq 1\right\}$ are WUOD, then for each $n \geq 1$ and any $v>0$ : 


$$
E \exp \left\{v \sum_{i=1}^{n} \xi_{i}\right\} \leq g_{u}(n) \prod E \exp v \xi_{i}
$$

In the following, we will use the assumptions that for any $\varepsilon>0$ Equation 2.2 and 2.3:

$$
\lim _{n \rightarrow \infty} \mathrm{g}_{U}(\mathrm{n}) e^{-\varepsilon n}=0
$$

And

$$
\lim _{n \rightarrow \infty} \mathrm{g}_{L}(\mathrm{n}) e^{-\varepsilon n}=0
$$

We define the sequence of the real value random variables $\left\{\xi_{n}, n \geq 1\right\}$ as Pairwise Quasi-Asymptotically Independent (PQAI) for any $i \neq j$ :

$$
\lim _{z \rightarrow \infty} P\left(\left|\xi_{i}\right| \wedge \xi_{j}>z \mid \xi_{i} \vee \xi_{j}>z\right)=0
$$

We also define the sequence of the real value random variables $\left\{\xi_{n}, n \geq 1\right\}$ as pairwise Strong QuasiAsymptotically Independent (pSQAI), for any $i \neq j$ :

$$
\lim _{z_{i} \wedge z_{j}} P\left(\left|\xi_{i}\right|>z_{i} \mid \xi_{j}>z_{j}\right)=0
$$

We list several corresponding results and remark the methods used in some papers mentioned above heavily rely on the i.i.d assumption on the claim size and the arrival times of successive claims. By observation and analysis, we compare the advantages and disadvantages of above dependent structures. The dependent case of WUOD and WLOD can allow some negatively and positively random variables. The PSQAI structure can include the WUOD and PQAI. In addition, when the random variables are nonnegative, the two structures of pSQAI and PQAI random variables are equivalent and the pSQAI structure is a more general dependent case than the WUOD. The ND structure is a relatively weak condition, the asymptotic behavior of the ruin probability is not sensitive to the ND structure.

\section{Result 1}

Theorem 1 of (Chen and $\mathrm{Ng}, 2007)$. Consider the renewal risk model in section 1 , if the claim sizes $\left\{X_{n}\right.$; $n \geq 1\}$ are pairwise ND with common distribution $H \in E R V$, the inter-arrival times $Y_{n}$ are i.i.d random variables and the $\{c(t), t \geq 0\}$ is a deterministic linear function and then the asymptotic for the ultimate ruin probability $\Phi(x)$ :

$$
\Phi(\mathrm{x}) \sim \int_{0}^{\infty}\left(\bar{H}\left(\mathrm{x} e^{\delta t^{\prime}}\right)\right) d \lambda\left(t^{\prime}\right)
$$

\section{Result 2}

Theorem 1 of Li et al. (2009). Consider the renewal risk model in Section1, if the claim sizes $\left\{X_{n} ; n \geq 1\right\}$ are pairwise NQD with common distribution $H \in D$, the interarrival times $\left\{Y_{n} ; n \geq 1\right\}$ are NLOD and the $\{c(t), t \geq 0\}$ is a deterministic linear function. In particular, if $H \in \mathcal{L}$ and $J_{H}^{-}>0$, we obtain the $\Phi(x, t)$ :

$$
\Phi(\mathrm{x}, \mathrm{t}) \sim \int_{0}^{t}\left(\bar{H}\left(\mathrm{x} e^{\delta t^{\prime}}\right)\right) d \lambda\left(t^{\prime}\right)
$$

\section{Result 3}

Theorem 1 of Shen and Lin (2008). Consider the renewal risk model, if the claim sizes $\left\{X_{n} ; n \geq 1\right\}$ are NOD random variables with common distribution $H \in L \cap D$, the inter-arrival times the inter-arrival times $\left\{Y_{n} ; n \geq 1\right\}$ are i.i.d with common exponential distribution $\{N(t), t \geq 0\}$ is a homogeneous Poisson process:

$$
\Phi(x, t) \sim \int_{0}^{t}\left(\bar{H}\left(\mathrm{x} e^{\delta t^{\prime}}\right)\right) d \lambda\left(t^{\prime}\right)
$$

\section{Result 4}

Theorem 1.1 of Wang et al. (2013). Consider the renewal risk model in Section 1. If the claim sizes $\left\{X_{n}\right.$; $n \geq 1\}$ are WUOD with common distribution $H \in \mathcal{L} \cap \mathcal{D}$, the inter-arrival times $\left\{Y_{n} ; n \geq 1\right\}$ are WLOD. Also holds the relations (2.11) and (2.12). Then for any finite $T \in \Lambda$, the relation (2.15) holds uniformly for $t \in \Lambda[0, T]$ and then we obtain the equivalent form for the $\Phi(x, T)$ :

$$
\Phi(\mathrm{x}, \mathrm{t}) \sim \int_{0}^{t}\left(\bar{H}\left(\mathrm{x} e^{\delta t^{\prime}}\right)\right) d \lambda\left(t^{\prime}\right)
$$

\section{Result 5}

Theorem 1.1 of Liu et al. (2012). Consider the renewal risk model in Section 1. If the claim sizes $\left\{X_{n}\right.$; $n \geq 1\}$ are UTAI with common distribution $H \in \mathcal{L} \cap \mathcal{D}$, the inter-arrival times $\left\{Y_{n} ; n \geq 1\right\}$ are WLOD such that the relation (2.11) holds. Then for any fixed $T \in \Lambda$, then:

$$
\Phi(\mathrm{x}, \mathrm{t}) \sim \int_{0}^{t}\left(\bar{H}\left(\mathrm{x} e^{\delta t^{\prime}}\right)\right) d \lambda\left(t^{\prime}\right)
$$




\section{Result 6}

Theorem 3.1 of Li (2013). Consider the by-claim model in Section 1, assuming that $\left\{X_{n}, X_{n} ; n \geq 1\right\}\left\{\theta_{n}\right.$; $n \geq 1\}$ and $\left\{T_{n} ; n \geq 1\right\}$ are mutually independent, $X_{1}, Y_{1}, X_{2}$, $Y_{2}$ are PQAI and random pairs $\left(X_{1}, Y_{1}\right),\left(X_{2}, Y_{2}\right) \ldots$ are identically distributed. Let distributed. Let $\bar{H} \in E R V$ and $\bar{F}$ also $\in E R V$, then we obtain:

$$
\begin{aligned}
& \Phi(\mathrm{x}, \mathrm{t}) \sim \int_{0}^{t}\left(\bar{H}\left(\mathrm{x} e^{\delta t^{\prime}}\right)\right) \lambda\left(t^{\prime}\right) \\
& +\int_{0}^{t} \int_{0}^{t-t^{\prime}}\left(\bar{F}\left(\mathrm{x} e^{\delta\left(s^{\prime}+t^{\prime}\right)}\right)\right) d G\left(s^{\prime}\right) d \lambda\left(t^{\prime}\right)
\end{aligned}
$$

For the risk models and results, we may discuss them in various aspects according to the motivation of research, such as the general risk model or renewal risk model, independent structure or dependent structure, some common heavy-tailed distribution classes, the constant interest rate or not and so on. By analysis, we found that the claim sizes and the inter-arrival times in results of (Li et al., 2009; Wang et al., 2013; Li, 2013) satisfied the different dependent structures, it is a stronger restriction than the i.i.d condition in result of Chen and $\mathrm{Ng}$ (2007). But among the different dependent structures, we may have different choices in different risk models and then lead to different results, such as in Liu et al. (2012), he required both the common distribution of claim sizes and inter-arrival times follow the intersection class, but in many cases, the author chose a more mild condition ERV. Furthermore, in terms of common distribution, some papers involves a more complicated case, in Li et al. (2009) paper and Yang and Wang (2010), he remarked the upper and lower Matuszewska index, we also consider the upper and lower Matuszewska index in the renewal risk model. But in Wang et al. (2013) paper, he canceled the condition $J_{H}{ }^{-}$In particular, in background section we introduced the relation (2.10) and (2.11), Wang et al. (2013) considered them in [Result 4] and we will discuss them in our renewal risk model. In addition, the [Result 2] to [Result 5] mainly investigate the asymptotic behavior of ruin probability in finite time and then the [Result 1] and [Result 6] worked on the formula of ultimate ruin probability in risk model. Generally speaking, the premium function $c(t)$ is a general stochastic process, but in some paper, it is assumed that the $c(t)$ is a deterministic linear function, such as in Li et al. (2009) and Chen and $\mathrm{Ng}$ (2007). Furthermore, we do not always require $\delta>0$ and the inter-arrival times may not have an exponential distribution, but in most cases we define that the $\delta$ is constant interest rate, sometimes $\delta$ yield 0 and the inter-arrival times may follow a common exponential distribution. Finally, we consider the $N(t)$ factor, in risk model section we define $N(t)$ to constitute a renewal counting process, but in the result of Shen and Lin (2008), the $N(t)$ is a homogeneous Poisson process, which follow the Poisson distribution with associated parameter $\lambda$.

\section{MAIN RESULTS}

In this study, we still investigate the renewal risk model and require the claim sizes and the inter-arrival times satisfy the pSQAI and WLOD structure. We can get a stronger result under mild assumption, in which the $H$ and $F$ belong to $\mathcal{L} \cap \mathcal{D}$. In addition, the risk model involving by-claim parts can also lead to a different result. So we have the following results:

\section{Lemma 3.1}

If $\mathrm{P}$ is a probability function of $A_{i}$ and $A_{j}$ are any set, then Equation 3.1:

$$
P\left(\bigcup_{i=1}^{m} A_{i}\right) \geq \sum_{i=1}^{m} P\left(A_{i}\right)-\sum_{1 \leq i \leq j \leq m} P\left(A_{i} A_{j}\right)
$$

\section{Proof}

We use mathematical induction to prove the relation (3.1). When $m=1$, we can easily draw the conclusion that Equation 3.2:

$$
P\left(A_{1}\right) \geq P\left(A_{1}\right)
$$

Assume that it is true that when $m=k$, i.e., Equation 3.3:

$$
P\left(\bigcup_{i=1}^{k} A_{i}\right) \geq \sum_{i=1}^{k} P\left(A_{i}\right)-\sum_{1 \leq i \leq j \leq k} P\left(A_{i} A_{j}\right)
$$

When $m=k+1$, we can use the basic probability formula $P(A \cup B)=P(A)+P(B)-P(A \cap B)$ Equation 3.4:

$$
\begin{aligned}
& P\left(\bigcup_{i=1}^{k+1} A_{i}\right)=P\left(\bigcup_{i=1}^{k} A_{i} \cup A_{k+1}\right) \\
& =P\left(\bigcup_{i=1}^{k} A_{i}\right)+P\left(A_{k+1}\right)-P\left(\left(\bigcup_{i=1}^{k} A_{i}\right) \cap\left(\left(A_{k+1}\right)\right)\right.
\end{aligned}
$$

Consequently, by induction assumption Equation 3.5:

$$
\begin{aligned}
& P\left(\bigcup_{i=1}^{k+1} A_{i}\right) \geq \sum_{i=1}^{k} P\left(A_{i}\right)-\sum_{1 \leq i \leq j \leq k} P\left(A_{i} A_{j}\right) \\
& +P\left(A_{k+1}\right)-P\left(\left(\bigcup_{i=1}^{k} A_{i}\right) \cap\left(\left(A_{k+1}\right)\right)\right.
\end{aligned}
$$


Therefore, combining the relation from (3.1) to (3.5), we finally get that Equation 3.6:

$$
P\left(\bigcup_{i=1}^{k+1} A_{i}\right) \geq \sum_{i=1}^{k+1} P\left(A_{i}\right)-\sum_{1 \leq i \leq j \leq k+1} P\left(A_{i} A_{j}\right)
$$

This ends the proof of the Lemma 3.1.

According to the Theorem 11 in Chapter 2 of (Kabanov et al., 1986), we have:

\section{Lemma 3.2 (Integration by parts)}

Suppose $f$ and $g$ are right continuous, non-decreasing and with left-hand limit functions on $[a, b]$, where $a<b<\mathbb{R}$. Then Equation 3.7:

$$
\int_{a}^{b} \mathrm{~g}(\mathrm{x}) d \mathrm{f}(\mathrm{x})=\mathrm{g}(\mathrm{b}) \mathrm{f}(\mathrm{b})-\mathrm{g}(\mathrm{a}) \mathrm{f}(\mathrm{a})-\int_{a}^{b} \mathrm{f}(\mathrm{x}-) d \mathrm{~g}(\mathrm{x})
$$

\section{Lemma 3.3}

Considering the renewal counting process $\{N(t), t \geq 0\}$ defined in (1.2). Suppose that $\left\{Y_{n}, n \geq 1\right\}$ satisfy the WLOD structure and then also holds the (2.12) relation. For any $T \in \Lambda$ and any $\gamma>0$, we obtain that Equation 3.8:

$$
\lim _{\mathrm{x} \rightarrow \infty} \sup _{\mathrm{t} \in \Lambda[0, \mathrm{~T}]} \lambda(\mathrm{t})^{-1} \mathrm{E}(\mathrm{N}(\mathrm{t}))^{\gamma} 1_{\{\mathrm{N}(\mathrm{t})>x\}}
$$

\section{Proof}

See the proof of Lemma 2.1 in Wang et al. (2013).

\section{Lemma 3.4}

Let $\left\{X_{n} ; n \geq 1\right\}$ and $\left\{Z_{n} ; n \geq 1\right\}$ be the mutual independent sequences with common distribution functions $H$ and $F$ belong to the class $\mathcal{L} \cap D$ uniformly for $t \in \Lambda[0, T], 1 \leq i \leq n, k=1$ or 2 .

Then Equation 3.9 and 10:

$$
\begin{aligned}
& P\left(X_{i} \mathrm{e}^{-\delta \omega_{\mathrm{i}}} 1_{\left\{\omega_{\mathrm{i}}<t\right\}}>((x \pm 2 l(x)) / k, N(t)=\mathrm{n})\right) \\
& \sim P\left(X_{i} \mathrm{e}^{-\delta \omega_{\mathrm{i}}} 1_{\left\{\omega_{\mathrm{i}}<t\right\}}>(x / k, N(t)=\mathrm{n})\right) \\
& P\left(Z_{i} \mathrm{e}^{-\delta\left(\omega_{\mathrm{i}}+T_{i}\right)} 1_{\left\{\omega_{\mathrm{i}}+T_{i}<t\right\}}>((x \pm 2 l(x)) / k, N(t)=\mathrm{n})\right) \\
& \sim P\left(Z_{i} \mathrm{e}^{-\delta\left(\omega_{\mathrm{i}}+T_{i}\right)} 1_{\left\{\omega_{\mathrm{i}}+T_{i}<t\right\}}>(x / k, N(t)=\mathrm{n})\right)
\end{aligned}
$$

\section{Lemma 3.5}

Under the assumption of Lemma 3.4, $X_{n}$ and $Z_{n}$ be the mutual independent sequences with common distribution functions $H$ and $F$ belong to the $\mathcal{L} \cap \mathcal{D}$ and both satisfy the pSQAI structure. For any $1 \leq i \leq n$, it holds uniformly $t \in \Lambda[0, T]$ :

$$
\begin{gathered}
\liminf _{\operatorname{xinf}_{\mathrm{t} \rightarrow \infty[0, \mathrm{~T}]}} \frac{P\left(X_{i} \mathrm{e}^{-\delta \omega_{\mathrm{i}}} 1_{\left\{\omega_{\mathrm{i}}<t\right\}}+Z_{i} \mathrm{e}^{-\delta\left(\omega_{\mathrm{i}}+T_{i}\right)} 1_{\left\{\omega_{\mathrm{i}}+T_{i}<t\right\}}\right.}{P\left(X_{i} \mathrm{e}^{-\delta \omega_{\mathrm{i}}} 1_{\left\{\omega_{\mathrm{i}}<t\right\}}>x, N(t)=n\right)} \geq 1 \\
+P\left(Z_{i} \mathrm{e}^{-\delta\left(\omega_{\mathrm{i}}+T_{i}\right)} 1_{\left\{\omega_{\mathrm{i}}+T_{i}<t\right\}}>x, N(t)=n\right)
\end{gathered}
$$

\section{Proof}

By the Lemma 3.1 and the formula $P(A \cup B) \leq P(A)+P(B)$, we can easily found:

$$
\begin{aligned}
& P\left(X_{i} \mathrm{e}^{-\delta \omega_{\mathrm{i}}} 1_{\left\{\omega_{\mathrm{i}}<t\right\}}+Z_{i} \mathrm{e}^{-\delta\left(\omega_{\mathrm{i}}+T_{i}\right)} 1_{\left\{\omega_{\mathrm{i}}+T_{i}<t\right\}}>x+l(x), N(t)=n\right) \\
& \geq P\left(\left(X_{i} \mathrm{e}^{-\delta \omega_{\mathrm{i}}} 1_{\left\{\omega_{\mathrm{i}}<t\right\}}>x+l(x), N(t)=n\right)\right. \\
& \left.\cup\left(Z_{i} \mathrm{e}^{-\delta\left(\omega_{\mathrm{i}}+T_{i}\right)} 1_{\left\{\omega_{\mathrm{i}}+T_{i}<t\right\}}>x, N(t)=n\right)\right) \\
& =P\left(\left(X_{i} \mathrm{e}^{-\delta \omega_{\mathrm{i}}} 1_{\left\{\omega_{\mathrm{i}}<t\right\}}>x+l(x), N(t)=n\right)\right) \\
& +P\left(Z_{i} \mathrm{e}^{-\delta\left(\omega_{\mathrm{i}}+T_{i}\right)} 1_{\left\{\omega_{\mathrm{i}}+T_{i}<t\right\}}>x, N(t)=n\right) \\
& -P\left(\left(X_{i} \mathrm{e}^{-\delta \omega_{\mathrm{i}}} 1_{\left\{\omega_{\mathrm{i}}<t\right\}}>x+l(x), N(t)=n\right)\right. \\
& \left.\cap\left(Z_{i} \mathrm{e}^{-\delta\left(\omega_{\mathrm{i}}+T_{i}\right)} 1_{\left\{\omega_{\mathrm{i}}+T_{i}<t\right\}}>x, N(t)=n\right)\right) \\
& \geq P\left(\left(X_{i} \mathrm{e}^{-\delta \omega_{\mathrm{i}}} 1_{\left\{\omega_{\mathrm{i}}<t\right\}}>x+l(x), N(t)=n\right)\right) \\
& +P\left(Z_{i} \mathrm{e}^{-\delta\left(\omega_{\mathrm{i}}+T_{i}\right)} 1_{\left\{\omega_{\mathrm{i}}+T_{i}<t\right\}}>x, N(t)=n\right) \\
& -P\left(X_{i} \mathrm{e}^{-\delta \omega_{\mathrm{i}}} 1_{\left\{\omega_{\mathrm{i}}<t\right\}}>x+l(x),\right. \\
& \left.Z_{i} \mathrm{e}^{-\delta\left(\omega_{\mathrm{i}}+T_{i}\right)} 1_{\left\{\omega_{\mathrm{i}}+T_{i}<t\right\}}>x, N(t)=n\right)
\end{aligned}
$$

From the last step, because of the fact that the independent relationship among $X_{i}, Z_{i}$ and $\omega_{i}$, we conclude:

$$
\begin{aligned}
& P\left(X_{i} \mathrm{e}^{-\delta \omega_{\mathrm{i}}} 1_{\left\{\omega_{\mathrm{i}}<t\right\}}>x+l(x), Z_{i}>x, N(t)=n\right) \\
& =P\left(X_{i} \mathrm{e}^{-\delta \omega_{\mathrm{i}}} 1_{\left\{\omega_{\mathrm{i}}<t\right\}}>x+l(x), N(t)=n\right) P\left(Z_{i}>x, N(t)=n\right)
\end{aligned}
$$

Because the basic property of probability and the $l(x)$ is large enough:

$$
\lim _{\mathrm{x} \rightarrow \infty} P\left(Z_{i}>x, N(t)=n\right)=0
$$

\section{Lemma 3.6}

Let $X_{n}$ and $Z_{n}$ be the mutual independent sequences with common distribution functions $H$ and $F$ belong to the $\mathcal{L} \cap \mathcal{D}$ and both sat and both satisfy the pSQAI structure. For any $1 \leq i \leq n$, it holds uniformly $t \in \Lambda[0, T]$ Equation 3.11: 
$\lim _{\mathrm{x} \rightarrow \infty} \sup _{\operatorname{t\in } \in \Lambda[0, \mathrm{~T}]} \sup _{P\left(X_{i} \mathrm{e}^{-\delta \omega_{\mathrm{i}}} 1_{\left\{\omega_{\mathrm{i}}<t\right\}}>x, N(t)=n\right)+P\left(Z_{i} \mathrm{e}^{-\delta\left(\omega_{\mathrm{i}}+T_{i}\right)} 1_{\left\{\omega_{\mathrm{i}}+T_{i}<t\right\}}>x, N(t)=n\right)} \leq 1$

For the convenience of proof, we define that:

$$
\begin{aligned}
& J=P\left(X_{i} \mathrm{e}^{-\delta \omega_{\mathrm{i}}} 1_{\left\{\omega_{\mathrm{i}}<t\right\}}+Z_{i} \mathrm{e}^{-\delta\left(\omega_{\mathrm{i}}+T_{i}\right)} 1_{\left\{\omega_{\mathrm{i}}+T_{i}<t\right\}}>x-l(x), N(t)=n\right) \\
& J^{*}=P\left(X_{i} \mathrm{e}^{-\delta \omega_{\mathrm{i}}} 1_{\left\{\omega_{\mathrm{i}}<t\right\}}>x, N(t)=n\right)+P\left(Z_{i} \mathrm{e}^{-\delta\left(\omega_{\mathrm{i}}+T_{i}\right)} 1_{\left\{\omega_{\mathrm{i}}+T_{i}<t\right\}}>x, N(t)=n\right) \\
& \mathrm{A}=\left\{X_{i} \mathrm{e}^{-\delta \omega_{\mathrm{i}}} 1_{\left\{\omega_{\mathrm{i}}<t\right\}}+Z_{i} \mathrm{e}^{-\delta\left(\omega_{\mathrm{i}}+T_{i}\right)} 1_{\left\{\omega_{\mathrm{i}}+T_{i}<t\right\}}>x-l(x)\right\} \\
& \left.B=\left\{X_{i} \mathrm{e}^{-\delta \omega_{\mathrm{i}}} 1_{\left\{\omega_{\mathrm{i}}<t\right\}}>x-2 l(x), N(t)=n\right\} \cup\left\{Z_{i} \mathrm{e}^{-\delta\left(\omega_{\mathrm{i}}+T_{i}\right)} 1_{\left\{\omega_{\mathrm{i}}+T_{i}<t\right\}}>x-2 l(x), N(t)=n\right)\right\}
\end{aligned}
$$

That is to say:

$$
\lim _{\mathrm{x} \rightarrow \infty} \sup \sup _{\mathrm{t} \in \Lambda[0, \mathrm{~T}]} \frac{J}{J^{*}} \leq 1
$$

\section{Proof}

Firstly, if $x$ is large enough, we consider the definition of $l(x)$ and the formula $P(A)=P(A B)+P\left(A B^{c}\right)$, it is obviously true:

$$
\begin{aligned}
& J \leq P\left(X_{i} \mathrm{e}^{-\delta \omega_{\mathrm{i}}} 1_{\left\{\omega_{\mathrm{i}}<t\right\}}+Z_{i} \mathrm{e}^{-\delta\left(\omega_{\mathrm{i}}+T_{i}\right)} 1_{\left\{\omega_{\mathrm{i}}+T_{i}<t\right\}}>x-l(x),\right. \\
& \left.\left(X_{i} \mathrm{e}^{-\delta \omega_{\mathrm{i}}} 1_{\left\{\omega_{\mathrm{i}}<t\right\}}>x-2 l(x), N(t)=n\right)\right) \\
& \left.\cup\left(Z_{i} \mathrm{e}^{-\delta\left(\omega_{\mathrm{i}}+T_{i}\right)} 1_{\left\{\omega_{\mathrm{i}}+T_{i}<t\right\}}>x-2 l(x), N(t)=n\right)\right) \\
& +P\left(X_{i} \mathrm{e}^{-\delta \omega_{\mathrm{i}}} 1_{\left\{\omega_{\mathrm{i}}<t\right\}}+Z_{i} \mathrm{e}^{-\delta\left(\omega_{\mathrm{i}}+T_{i}\right)} 1_{\left\{\omega_{\mathrm{i}}+T_{i}<t\right\}}>x-l(x),\right. \\
& \left(X_{i} \mathrm{e}^{-\delta \omega_{\mathrm{i}}} 1_{\left\{\omega_{\mathrm{i}}<t\right\}} \leq x-2 l(x),\right. \\
& \left.\left.Z_{i} \mathrm{e}^{-\delta\left(\omega_{\mathrm{i}}+T_{i}\right)} 1_{\left\{\omega_{\mathrm{i}}+T_{i}<t\right\}} \leq x-2 l(x), N(t)=n\right)\right)
\end{aligned}
$$

Followed by the above inequality, we apply the simple formula that $P\left(A \bigcup_{i=1}^{n} B\right) \leq P\left(\bigcup_{i=1}^{n} B\right) \leq \sum_{i=1}^{n} P(B)$ and Boole's inequality, we find:

$$
\begin{aligned}
& J \leq P\left(X_{i} \mathrm{e}^{-\delta \omega_{\mathrm{i}}} 1_{\left\{\omega_{\mathrm{i}}<t\right\}}>x-2 l(x), N(t)=n\right) \\
& +P\left(Z_{i} \mathrm{e}^{-\delta\left(\omega_{\mathrm{i}}+T_{i}\right)} 1_{\left\{\omega_{\mathrm{i}}+T_{i}<t\right\}}>x-2 l(x), N(t)=n\right) \\
& +P\left(X_{i} \mathrm{e}^{-\delta \omega_{\mathrm{i}}} 1_{\left\{\omega_{\mathrm{i}}<t\right\}}>\frac{x-l(x)}{2}\right. \\
& \left.\left.Z_{i} \mathrm{e}^{-\delta\left(\omega_{\mathrm{i}}+T_{i}\right)} 1_{\left\{\omega_{\mathrm{i}}+T_{i}<t\right\}}>l(x), N(t)=n\right)\right) \\
& +P\left(Z_{i} \mathrm{e}^{-\delta\left(\omega_{\mathrm{i}}+T_{i}\right)} 1_{\left\{\omega_{\mathrm{i}}+T_{i}<t\right\}}>\frac{x-l(x)}{2}\right. \\
& \left.\left.X_{i} \mathrm{e}^{-\delta \omega_{\mathrm{i}}} 1_{\left\{\omega_{\mathrm{i}}<t\right\}}>l(x), N(t)=n\right)\right)
\end{aligned}
$$

Considering the fact that $e^{-\delta(\omega i+T i)}$ and $e^{-\delta \omega i}$ belong to the interval $\left[e^{-\delta t}, 1\right]$, we may conclude that the set $\left\{X_{i} \mathrm{e}^{-\delta \omega_{\mathrm{i}}} 1_{\left\{\omega_{\mathrm{i}}<t\right\}}>\frac{x-l(x)}{2}, Z_{i} \mathrm{e}^{-\delta\left(\omega_{\mathrm{i}}+T_{i}\right)} 1_{\left\{\omega_{\mathrm{i}}+T_{i}<t\right\}}>l(x)\right\}$ $\subseteq\left\{X_{i} \mathrm{e}^{-\delta \omega_{\mathrm{i}}} 1_{\left\{\omega_{\mathrm{i}}<t\right\}}>\frac{x-l(x)}{2}, Z_{i}>l(x)\right\} \quad$ and the set $\left\{Z_{i} \mathrm{e}^{-\delta\left(\omega_{\mathrm{i}}+T_{i}\right)} 1_{\left\{\omega_{\mathrm{i}}+T_{i}<t\right\}}>\frac{x-l(x)}{2}, X_{i} \mathrm{e}^{-\delta \omega_{\mathrm{i}}} 1_{\left\{\omega_{\mathrm{i}}<t\right\}}>l(x)\right\}$ $\subseteq\left\{Z_{i} \mathrm{e}^{-\delta\left(\omega_{\mathrm{i}}+T_{i}\right)} 1_{\left\{\omega_{\mathrm{i}}+T_{i}<t\right\}}>\frac{x-l(x)}{2}, X_{i}>l(x)\right\}$

So we can apply the common probability formula if $\mathrm{A} \subseteq \mathrm{B}$, then $P(A)<P(B)$ to get the following expression:

$$
\begin{aligned}
& J \leq P\left(X_{i} \mathrm{e}^{-\delta \omega_{\mathrm{i}}} 1_{\left\{\omega_{\mathrm{i}}<t\right\}}>x-2 l(x), N(t)=n\right) \\
& +P\left(Z_{i} \mathrm{e}^{-\delta\left(\omega_{\mathrm{i}}+T_{i}\right)} 1_{\left\{\omega_{\mathrm{i}}+T_{i}<t\right\}}>x-2 l(x), N(t)=n\right) \\
& \left.+P\left(X_{i} \mathrm{e}^{-\delta \omega_{\mathrm{i}}} 1_{\left\{\omega_{\mathrm{i}}<t\right\}}>\frac{x-l(x)}{2}, Z_{i}>l(x), N(t)=n\right)\right) \\
& +P\left(Z_{i} \mathrm{e}^{-\delta\left(\omega_{\mathrm{i}}+T_{i}\right)} 1_{\left\{\omega_{\mathrm{i}}+T_{i}<t\right\}}>\frac{x-l(x)}{2}, X_{i}>l(x), N(t)=n\right)
\end{aligned}
$$

By the independent relationship among the $X_{i}, Z_{i}$ and $\left\{\omega_{i}, T_{i}\right\}$, we have:

$$
\begin{aligned}
& J \leq P\left(X_{i} e^{\left(-? ?_{i}\right)} 1_{?_{i}<t}>x-2 l(x), N(t)=n\right) \\
& +P\left(Z_{i} \mathrm{e}^{-\delta\left(\omega_{\mathrm{i}}+T_{i}\right)} 1_{\left\{\omega_{\mathrm{i}}+T_{i}<t\right\}}>x-2 l(x), N(t)=n\right) \\
& +P\left(X_{i} \mathrm{e}^{-\delta \omega_{\mathrm{i}}} 1_{\left\{\omega_{\mathrm{i}}<t\right\}}>\frac{x-l(x)}{2}, N(t)=n\right) P\left(Z_{i}>l(x)\right) \\
& +P\left(Z_{i} \mathrm{e}^{-\delta\left(\omega_{\mathrm{i}}+T_{i}\right)} 1_{\left\{\omega_{\mathrm{i}}+T_{i}<t\right\}}>\frac{x-l(x)}{2}, N(t)=n\right) P\left(X_{i}>l(x)\right)
\end{aligned}
$$
get:

By the property of class $D$ and Lemma 3.4 , we can 


$$
\begin{aligned}
& P\left(X_{i} \mathrm{e}^{-\delta \omega_{\mathrm{i}}} 1_{\left\{\omega_{\mathrm{i}}<t\right\}}>\frac{x-l(x)}{2}, N(t)=n\right) \\
& \sim P\left(X_{i} \mathrm{e}^{-\delta \omega_{\mathrm{i}}} 1_{\left\{\omega_{\mathrm{i}}<t\right\}}>x-l(x), N(t)=n\right) \\
& \sim P\left(X_{i} \mathrm{e}^{-\delta \omega_{\mathrm{i}}} 1_{\left\{\omega_{\mathrm{i}}<t\right\}}>x, N(t)=n\right) \\
& P\left(Z_{i} \mathrm{e}^{-\delta\left(\omega_{\mathrm{i}}+T_{i}\right)} 1_{\left\{\omega_{\mathrm{i}}+T_{i}<t\right\}}>\frac{x-l(x)}{2}, N(t)=n\right) \\
& \sim P\left(Z_{i} \mathrm{e}^{-\delta\left(\omega_{\mathrm{i}}+T_{i}\right)} 1_{\left\{\omega_{\mathrm{i}}+T_{i}<t\right\}}>x-l(x), N(t)=n\right) \\
& \sim P\left(Z_{i} \mathrm{e}^{-\delta\left(\omega_{\mathrm{i}}+T_{i}\right)} 1_{\left\{\omega_{\mathrm{i}}+T_{i}<t\right\}}>x, N(t)=n\right)
\end{aligned}
$$

Thus, if $x$ is large enough:

$$
\begin{aligned}
& \lim _{\mathrm{x} \rightarrow \infty} P\left(X_{i} \mathrm{e}^{-\delta \omega_{\mathrm{i}}} 1_{\left\{\omega_{\mathrm{i}}<t\right\}}>\frac{x-l(x)}{2}, N(t)=n\right) P\left(Z_{i}>l(x)\right)=0 \\
& \lim _{\mathrm{x} \rightarrow \infty} P\left(Z_{i} \mathrm{e}^{-\delta\left(\omega_{\mathrm{i}}+T_{i}\right)} 1_{\left\{\omega_{\mathrm{i}}+T_{i}<t\right\}}\right. \\
& \left.>\frac{x-l(x)}{2}, N(t)=n\right) P\left(X_{i}>l(x)\right)=0
\end{aligned}
$$

Hence, we may find:

$$
\lim _{\mathrm{x} \rightarrow \infty} \sup \sup _{\mathrm{t} \in \Lambda[0, \mathrm{~T}]} \frac{J}{J^{*}} \leq 1
$$

Consequently, we hold the relation (3.12).

This ends the proof of Lemma (3.6).

\section{Lemma 3.7}

For the renewal risk model introduced in Risk Theory section, we have:

$$
\begin{aligned}
& \sum_{\mathrm{k}=1}^{\infty} P\left(X_{k} \mathrm{e}^{-\delta \omega_{\mathrm{k}}} 1_{\left\{\omega_{\mathrm{k}}<t\right\}}>x, N(t) \geq k\right) \\
& +P\left(Z_{k} \mathrm{e}^{-\delta\left(\omega_{\mathrm{k}}+T_{k}\right)} 1_{\left\{\omega_{\mathrm{k}}+T_{k}<t\right\}}>x, N(t) \geq k\right) \\
& =\int_{0}^{t} \bar{H}\left(x e^{\delta t^{\prime}}\right) d \lambda\left(t^{\prime}\right)+\int_{0}^{t} \int_{0}^{\mathrm{t}-\mathrm{t}^{\prime}} \bar{F}\left(\mathrm{xe}^{\delta\left(\mathrm{t}^{\prime}+\mathrm{s}^{\prime}\right)}\right) d G\left(s^{\prime}\right) d \lambda\left(t^{\prime}\right)
\end{aligned}
$$

\section{Proof}

Firstly, we should define the common distribution of $\omega_{k}$ as $V_{\omega_{k}}(t)$ Equation 3.12:

$$
\begin{aligned}
& P\left(X_{k} \mathrm{e}^{-\delta \omega_{\mathrm{k}}} 1_{\left\{\omega_{\mathrm{k}}<t\right\}}>x, N(t) \geq k\right) \\
& +P\left(Z_{k} \mathrm{e}^{-\delta\left(\omega_{\mathrm{k}}+T_{k}\right)} 1_{\left\{\omega_{\mathrm{k}}+T_{k}<t\right\}}>x, N(t) \geq k\right) \\
& =\int_{0}^{t} \bar{H}\left(\mathrm{xe}^{\delta \mathrm{t}^{\prime}}\right) d V_{\omega_{\mathrm{k}}}\left(t^{\prime}\right) \\
& +\int_{0}^{t} \int_{0}^{t-t^{\prime}} \bar{F}\left(\mathrm{xe}^{\delta\left(\mathrm{t}^{\prime}+\mathrm{s}^{\prime}\right)}\right) d G\left(s^{\prime}\right) d V_{\omega_{k}}\left(t^{\prime}\right)
\end{aligned}
$$

According to the non-decreasing condition in Lemma 3.2 , we reorganize the relation (3.13):

$$
\begin{aligned}
& P\left(X_{k} \mathrm{e}^{-\delta \omega_{\mathrm{k}}} 1_{\left\{\omega_{\mathrm{k}}<t\right\}}>x, N(t) \geq k\right) \\
& +P\left(Z_{k} \mathrm{e}^{-\delta\left(\omega_{\mathrm{k}}+T_{k}\right)} 1_{\left\{\omega_{\mathrm{k}}+T_{k}<t\right\}}>x, N(t) \geq k\right) \\
& =\int_{0}^{t} 1-\mathcal{H}\left(\mathrm{xe}^{\delta \mathrm{t}^{\prime}}\right) d V_{\omega_{\mathrm{k}}}\left(t^{\prime}\right) \\
& +\int_{0}^{t} \int_{0}^{t-t^{\prime}} 1-F\left(\mathrm{xe}^{\delta\left(\mathrm{t}^{\prime}+\mathrm{s}^{\prime}\right)}\right) d G\left(s^{\prime}\right) d V_{\omega_{\mathrm{k}}}\left(t^{\prime}\right) \\
& =\left(V_{\omega_{\mathrm{k}}}(t)-V_{\omega_{\mathrm{k}}}(0)\right)-\int_{0}^{t} \mathcal{H}\left(\mathrm{xe}^{\delta \mathrm{t}^{\prime}}\right) d V_{\omega_{\mathrm{k}}}\left(t^{\prime}\right) \\
& -V_{\omega_{\mathrm{k}}}(0)(G(t)-G(0)) \\
& -\int_{0}^{t} \int_{0}^{t-t^{\prime}} F\left(\mathrm{xe}^{\delta\left(\mathrm{t}^{\prime}+\mathrm{s}^{\prime}\right)}\right) d G\left(s^{\prime}\right) d V_{\omega_{\mathrm{k}}}\left(t^{\prime}\right) \\
& =\left(V_{\omega_{\mathrm{k}}}(t)-V_{\omega_{\mathrm{k}}}(0)\right)-\int_{0}^{t} \mathcal{H}\left(\mathrm{xe}^{\delta \mathrm{t}^{\prime}}\right) d V_{\omega_{\mathrm{k}}}\left(t^{\prime}\right) \\
& -\mathcal{H}(x) V_{\omega_{\mathrm{k}}}(0)-V_{\omega_{\mathrm{k}}}(0)(G(t)-G(0)) \\
& -\int_{0}^{t} \int_{0}^{t-t^{\prime}} F\left(\mathrm{xe}^{\delta\left(\mathrm{t}^{\prime}+\mathrm{s}^{\prime}\right)}\right) d G\left(s^{\prime}\right) d V_{\omega_{\mathrm{k}}}\left(t^{\prime}\right) \\
& -\int_{0}^{t} F\left(\mathrm{xe}^{\delta\left(\mathrm{t}^{\prime}+\mathrm{s}^{\prime}\right)}\right) G(0) d V_{\omega_{\mathrm{k}}}\left(t^{\prime}\right) \\
& =\left(V_{\omega_{\mathrm{k}}}(t)-V_{\omega_{\mathrm{k}}}(0)\right)-\mathcal{H}\left(\mathrm{xe}^{\delta \mathrm{t}}\right) V_{\omega_{\mathrm{k}}}(\mathrm{t})+\mathcal{H}(x) V_{\omega_{\mathrm{k}}}(0) \\
& +\int_{0}^{t} V_{\omega_{\mathrm{k}}}\left(\mathrm{t}^{\prime}-\right) d \mathcal{H}\left(\mathrm{xe}^{\delta \mathrm{t}}\right)-\mathcal{H}(x) V_{\omega_{\mathrm{k}}}(0)-V_{\omega_{\mathrm{k}}}(0)(G(t)-G(0)) \\
& -\int_{0}^{t} F\left(\mathrm{xe}^{\delta \mathrm{t}}\right) G\left(t-t^{\prime}\right) d V_{\omega_{\mathrm{k}}}\left(t^{\prime}\right) \\
& +\int_{0}^{t} F\left(\mathrm{xe}^{\delta\left(\mathrm{t}^{\prime}+\mathrm{s}^{\prime}\right)}\right) G(0) d V_{\omega_{\mathrm{k}}}\left(t^{\prime}\right) \\
& +\int_{0}^{t} \int_{0}^{t-t^{\prime}} G\left(\mathrm{~s}^{\prime}\right) d F\left(\mathrm{xe}^{\delta\left(\mathrm{t}^{\prime}+\mathrm{s}^{\prime}\right)}\right) d V_{\omega_{\mathrm{k}}}\left(t^{\prime}\right) \\
& -\int_{0}^{t} F\left(\mathrm{xe}^{\delta(\mathrm{t}+\mathrm{s})}\right) G(0) d V_{\omega_{\mathrm{k}}}\left(t^{\prime}\right) \\
& \int_{0}^{t} V_{\omega_{\mathrm{k}}}\left(\mathrm{t}^{\prime}-\right) d H\left(\mathrm{xe}^{\delta \mathrm{t}}\right)-H\left(\mathrm{xe}^{\delta \mathrm{t}}\right) V_{\omega_{\mathrm{k}}}(\mathrm{t}) \\
& +\int_{0}^{t} \int_{0}^{t-t^{\prime}} G\left(\mathrm{~s}^{\prime}\right) d F\left(\mathrm{xe}^{\delta\left(\mathrm{t}^{\prime}+\mathrm{s}^{\prime}\right)}\right) d V_{\omega_{\mathrm{k}}}\left(t^{\prime}\right) \\
& -\int_{0}^{t} F\left(\mathrm{xe}^{\delta \mathrm{t}}\right) G\left(\mathrm{t}-\mathrm{t}^{\prime}\right) d V_{\omega_{\mathrm{k}}}\left(t^{\prime}\right)
\end{aligned}
$$

We should make clear that $V_{\omega_{\mathrm{k}}}\left(\mathrm{t}^{\prime}-\right)=P\left(\omega_{k}<t\right)$ so the $\omega_{k} \leq t$ is equivalent to the $N(t) \geq k$ and $\omega_{k}<t$ is equivalent to the $N(t)>k$, we can derive: 


$$
\sum_{\mathrm{k}=1}^{\infty} V_{\omega_{\mathrm{k}}}\left(\mathrm{t}^{\prime}\right)=\sum_{\mathrm{k}=1}^{\infty} P(N(t) \geq k)=E N(t)<\infty
$$

And:

$$
\sum_{\mathrm{k}=1}^{\infty} V_{\omega_{\mathrm{k}}}\left(\mathrm{t}^{\prime}-\right)=\sum_{\mathrm{k}=1}^{\infty} P(N(t-) \geq k)=E N(t-)<\infty
$$

In the risk model section, we have already shown that $E N(t)$ is a non-decreasing and right continuous function. According to the Lemma 3.2, we can easily obtain that:

$$
\begin{aligned}
& \sum_{\mathrm{k}=1}^{\infty}\left(\begin{array}{l}
P\left(X_{k} \mathrm{e}^{-\delta \omega_{\mathrm{k}}} 1_{\left\{\omega_{\mathrm{k}}<t\right\}}>x, N(t) \geq k\right) \\
+P\left(Z_{k} \mathrm{e}^{-\delta\left(\omega_{\mathrm{k}}+T_{k}\right)} 1_{\left\{\omega_{\mathrm{k}}+T_{k}<t\right\}}>x, N(t) \geq k\right)
\end{array}\right) \\
& =\int_{0}^{t} \sum_{\mathrm{k}=1}^{\infty} V_{\omega_{\mathrm{k}}}\left(\mathrm{t}^{\prime}-\right) d H\left(\mathrm{x}^{\delta \mathrm{t}}\right) \\
& -\mathcal{H}\left(\mathrm{xe}^{\delta \mathrm{t}}\right) \sum_{\mathrm{k}=1}^{\infty} V_{\omega_{\mathrm{k}}}\left(\mathrm{t}^{\prime}\right) \\
& +\int_{0}^{t} \int_{0}^{t-t^{\prime}} G(\mathrm{~s}) d F\left(\mathrm{xe}^{\delta\left(\mathrm{t}^{\prime}+\mathrm{s}^{\prime}\right)}\right) d \sum_{\mathrm{k}=1}^{\infty} V_{\omega_{\mathrm{k}}}\left(\mathrm{t}^{\prime}\right) \\
& -\int_{0}^{t} F\left(\mathrm{xe}^{\delta \mathrm{t}}\right) G\left(\mathrm{t}-\mathrm{t}^{\prime}\right) \sum_{\mathrm{k}=1}^{\infty} V_{\omega_{\mathrm{k}}}\left(\mathrm{t}^{\prime}\right) \\
& =\int_{0}^{t} E N(t-) d H\left(\mathrm{xe}^{\delta \mathrm{t}}\right)+\bar{H}\left(\mathrm{xe}^{\delta \mathrm{t}}\right) E N(t) \\
& +\int_{0}^{t} \int_{0}^{t-t^{\prime}} G\left(\mathrm{~s}^{\prime}\right) d F\left(\mathrm{xe}^{\delta\left(\mathrm{t}^{\prime}+\mathrm{s}^{\prime}\right)}\right) d E N(t) \\
& +\bar{F}\left(\mathrm{xe}^{\delta \mathrm{t}}\right) G\left(\mathrm{t}-\mathrm{t}^{\prime}\right)(E N(t) E N(0))
\end{aligned}
$$

We should apply the integration by parts again. We get:

$$
\begin{aligned}
& \sum_{\mathrm{k}=1}^{\infty}\left(\begin{array}{l}
P\left(X_{k} \mathrm{e}^{-\delta \omega_{\mathrm{k}}} 1_{\left\{\omega_{\mathrm{k}}<t\right\}}>x, N(t) \geq k\right) \\
+P\left(Z_{k} \mathrm{e}^{-\delta\left(\omega_{\mathrm{k}}+T_{k}\right)} 1_{\left\{\omega_{\mathrm{k}}+T_{k}<t\right\}}>x, N(t) \geq k\right)
\end{array}\right) \\
& =\int_{0}^{t} \lambda(t-) d H\left(\mathrm{xe}^{\delta \mathrm{t}}\right) \int_{0}^{t} \int_{0}^{t-t^{\prime}} G\left(\mathrm{~s}^{\prime}\right) d \bar{F}\left(\mathrm{xe}^{\delta\left(\mathrm{t}^{\prime}+\mathrm{s}^{\prime}\right)}\right) d \lambda(t) \\
& =H\left(\mathrm{xe}^{\delta \mathrm{t}}\right) E N(t)-\int_{0}^{t} H\left(\mathrm{xe}^{\delta \mathrm{t}}\right) d \lambda(t) \\
& +\bar{F}\left(\mathrm{xe}^{\delta \mathrm{t}}\right) G\left(\mathrm{t}-\mathrm{t}^{\prime}\right)(E N(t) E N(0)) \\
& -\int_{0}^{t} \int_{0}^{t} F\left(\mathrm{xe}^{\delta\left(\mathrm{t}^{\prime}+\mathrm{s}^{\prime}\right)}\right) d G\left(\mathrm{~s}^{\prime}\right) d \lambda\left(t^{\prime}\right)
\end{aligned}
$$

Therefore:

$$
\begin{aligned}
& \sum_{\mathrm{k}=1}^{\infty}\left(\begin{array}{l}
P\left(X_{k} \mathrm{e}^{-\delta \omega_{\mathrm{k}}} 1_{\left\{\omega_{\mathrm{k}}<t\right\}}>x, N(t) \geq k\right) \\
+P\left(Z_{k} \mathrm{e}^{-\delta\left(\omega_{\mathrm{k}}+T_{k}\right)} 1_{\left\{\omega_{\mathrm{k}}+T_{k}<t\right\}}>x, N(t) \geq k\right)
\end{array}\right) \\
& =\int_{0}^{t} \bar{H}\left(\mathrm{xe}^{\delta \mathrm{t}}\right) d \lambda(t)+\int_{0}^{t} \int_{0}^{t-t^{\prime}} \bar{F}\left(\mathrm{xe}^{\delta\left(\mathrm{t}^{\prime}+\mathrm{s}^{\prime}\right)}\right) d G\left(\mathrm{~s}^{\prime}\right) d \lambda\left(\mathrm{t}^{\prime}\right)
\end{aligned}
$$

It ends the proof of Lemma (3.7).

Our main result is for the approximation of finite ruin probability of the renewal risk model with constant interest rate. In the following section, we will give proofs of some related Lemmas mentioned in the following section.

Lemma 3.8 Consider the risk model in Section 1. Assume that the $\left\{X_{n}, Y_{n} ; n \geq 1\right\},\left\{\omega_{n} ; n \geq 1\right\}$ and $\left\{T_{n} ; n \geq 1\right\}$ are mutually independent. Let claim size $\left\{X_{n} ; n \geq 1\right\}$ and by-claim parts $\left\{Z_{n} ; n \geq 1\right\}$ be pSQAI dependent structure with common distribution functions $\bar{H}$ and $\bar{F}$ belong to the $\mathcal{L} \cap \mathcal{D}$ and the inter-arrival time $\left\{Y_{n} ; n \geq 1\right\}$ be WLOD with common distribution $V$ and also hold the relation (2.11) and (2.12). Then for any fixed $T \in \Lambda$, it holds uniformly $t \in \Lambda[0, \mathrm{~T}]$ :

$$
\begin{aligned}
& \Phi(\mathrm{x}, \mathrm{t}) \sim \int_{0}^{t}\left(\bar{H}\left(\mathrm{x} e^{\delta t^{\prime}}\right)\right) d \lambda\left(t^{\prime}\right) \\
& +\int_{0}^{t} \int_{0}^{t-t^{\prime}}\left(\bar{F}\left(\mathrm{x} e^{\delta\left(s^{\prime}+t^{\prime}\right)}\right)\right) d G\left(s^{\prime}\right) d \lambda\left(t^{\prime}\right)
\end{aligned}
$$

i.e.:

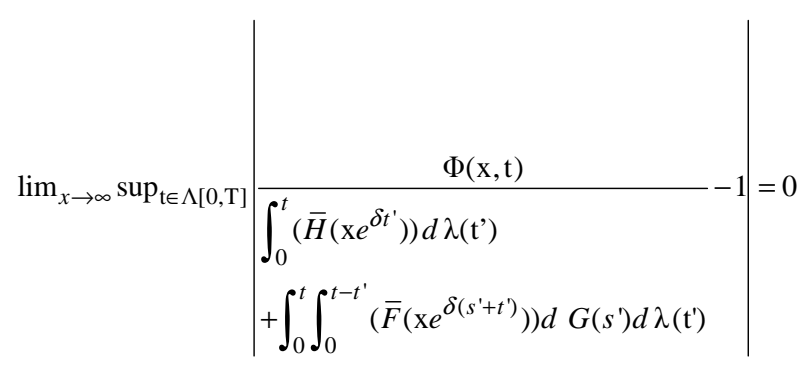

\section{PROOFS}

\section{Lemma 4.1}

Consider the risk model introduced in Section 1. Let $X_{n}$ be the sequence of pSQAI structure random variables with a common distribution function of $H(x)$ and $Z_{n}$ be the sequence of pSQAI structure random variables with a common distribution function of $F(x)$. The $X_{n}$ and $Z_{n}$ are $H(x) \in \mathcal{L} \cap \mathcal{D}$ and $F(x) \in \mathcal{L} \cap \mathcal{D}$ for any fixed $\mathrm{n}$, it holds uniformly $t \in \Lambda[0, T]$ Equation 4.1: 


$$
\begin{aligned}
& P\left(\sum_{\mathrm{k}=1}^{\infty}\left(X_{k} \mathrm{e}^{-\delta \omega_{\mathrm{k}}} 1_{\left\{\omega_{\mathrm{k}}<t\right\}}+Z_{k} \mathrm{e}^{-\delta\left(\omega_{\mathrm{k}}+T_{k}\right)} 1_{\left\{\omega_{\mathrm{k}}+T_{k}<t\right\}}>x\right), N(t)=n\right) \\
& \sim \sum_{\mathrm{k}=1}^{\infty} P\left(X_{k} \mathrm{e}^{-\delta \omega_{\mathrm{k}}} 1_{\left\{\omega_{\mathrm{k}}<t\right\}}>x, N(t)=n\right)+\sum_{\mathrm{k}=1}^{\infty} P\left(Z_{k} \mathrm{e}^{-\delta\left(\omega_{\mathrm{k}}+T_{k}\right)} 1_{\left\{\omega_{\mathrm{k}}+T_{k}<t\right\}}>x, N(t)=n\right)
\end{aligned}
$$

Suppose that:

$$
\begin{aligned}
& \hat{J}=P\left(\sum_{\mathrm{k}=1}^{\infty}\left(X_{k} \mathrm{e}^{-\delta \omega_{\mathrm{k}}} 1_{\left\{\omega_{\mathrm{k}}<t\right\}}+Z_{k} \mathrm{e}^{-\delta\left(\omega_{\mathrm{k}}+T_{k}\right)} 1_{\left\{\omega_{\mathrm{k}}+T_{k}<t\right\}}>x\right), N(t)=n\right) \\
& \hat{J}=\sum_{\mathrm{k}=1}^{\infty} P\left(X_{k} \mathrm{e}^{-\delta \omega_{\mathrm{k}}} 1_{\left\{\omega_{\mathrm{k}}<t\right\}}>x, N(t)=n\right)+\sum_{\mathrm{k}=1}^{\infty} P\left(Z_{k} \mathrm{e}^{-\delta\left(\omega_{\mathrm{k}}+T_{k}\right)} 1_{\left\{\omega_{\mathrm{k}}+T_{k}<t\right\}}>x, N(t)=n\right)
\end{aligned}
$$

Which is understood as Equation 4.2:

$$
\lim _{\mathrm{x} \rightarrow \infty} \sup _{\mathrm{t} \in \Lambda[0, \mathrm{~T}]}\left|\frac{\hat{J}}{\tilde{J}}-1\right|=0
$$

\section{Proof}

We need to prove the two inequalities Equation 4.3 and 4.4:

$$
\lim _{\mathrm{x} \rightarrow \infty} \sup _{\mathrm{t} \in \Lambda[0, \mathrm{~T}]} \frac{\hat{J}}{\tilde{J}} \leq 1
$$

$$
\lim _{\mathrm{x} \rightarrow \infty} \inf \inf _{\mathrm{t} \in \Lambda[0, \mathrm{~T}]} \frac{\hat{J}}{\tilde{J}} \geq 1
$$

Firstly, we are ready to prove the relation (4.4), recalling the definition of the $l(x)$ satisfying the (2.7) and Lemma 3.1, we have Equation 4.5:

$$
\begin{aligned}
& \hat{J} \geq \sum_{i=1}^{n} P\left(\sum_{\mathrm{k}=1}^{n}\left(\begin{array}{l}
X_{k} \mathrm{e}^{-\delta \omega_{\mathrm{k}}} 1_{\left\{\omega_{\mathrm{k}}<t\right\}} \\
+Z_{k} \mathrm{e}^{-\delta\left(\omega_{\mathrm{k}}+T_{k}\right)} 1_{\left\{\omega_{\mathrm{k}}+T_{k}<t\right\}}>x
\end{array}\right),\right. \\
& \left(X_{i} \mathrm{e}^{-\delta \omega_{\mathrm{i}}} 1_{\left\{\omega_{\mathrm{i}}<t\right\}}+Z_{i} \mathrm{e}^{-\delta\left(\omega_{i}+T_{i}\right)} 1_{\left\{\omega_{\mathrm{i}}+T_{i}<t\right\}}>x\right. \\
& +l(x), N(t)=n) \\
& -\sum_{1 \leq \mathrm{k} \leq \mathrm{n}, \mathrm{k} \neq \mathrm{i}}\left(\begin{array}{l}
X_{k} \mathrm{e}^{-\delta \omega_{\mathrm{k}}} 1_{\left\{\omega_{\mathrm{k}}<t\right\}} \\
+Z_{k} \mathrm{e}^{-\delta\left(\omega_{\mathrm{k}}+T_{k}\right)} 1_{\left\{\omega_{\mathrm{k}}+T_{k}<t\right\}}>x+l(x)
\end{array}\right), \\
& \left(X_{i} \mathrm{e}^{-\delta \omega_{\mathrm{i}}} 1_{\left\{\omega_{\mathrm{i}}<t\right\}}+Z_{i} \mathrm{e}^{-\delta\left(\omega_{i}+T_{i}\right)} 1_{\left\{\omega_{\mathrm{i}}+T_{i}<t\right\}}\right. \\
& >x+l(x), N(t)=n)
\end{aligned}
$$

By observation, we add the inequality:

$$
\begin{gathered}
\sum_{1 \leq \mathrm{k} \leq \mathrm{n}, \mathrm{k} \neq \mathrm{i}}\left(\begin{array}{l}
X_{k} \mathrm{e}^{-\delta \omega_{\mathrm{k}}} 1_{\left\{\omega_{\mathrm{k}}<t\right\}} \\
+Z_{k} \mathrm{e}^{-\delta\left(\omega_{\mathrm{k}}+T_{k}\right)} 1_{\left\{\omega_{\mathrm{k}}+T_{k}<t\right\}}>-l(x)
\end{array}\right) \\
\text { tothe }\left(\begin{array}{l}
X_{i} \mathrm{e}^{-\delta \omega_{\mathrm{i}}} 1_{\left\{\omega_{\mathrm{i}}<t\right\}}+Z_{i} \mathrm{e}^{-\delta\left(\omega_{i}+T_{i}\right)} 1_{\left\{\omega_{\mathrm{i}}+T_{i}<t\right\}} \\
>x+l(x), N(t)=n
\end{array}\right)
\end{gathered}
$$

Thus, we get:

$$
\begin{aligned}
& P\left(\sum_{\mathrm{k}=1}^{n}\left(\begin{array}{l}
X_{k} \mathrm{e}^{-\delta \omega_{\mathrm{k}}} 1_{\left\{\omega_{\mathrm{k}}<t\right\}} \\
+Z_{k} \mathrm{e}^{-\delta\left(\omega_{\mathrm{k}}+T_{k}\right)} 1_{\left\{\omega_{\mathrm{k}}+T_{k}<t\right\}}>x
\end{array}\right),\right. \\
& \left(\begin{array}{l}
X_{i} \mathrm{e}^{-\delta \omega_{\mathrm{i}}} 1_{\left\{\omega_{\mathrm{i}}<t\right\}}+Z_{i} \mathrm{e}^{-\delta\left(\omega_{i}+T_{i}\right)} 1_{\left\{\omega_{\mathrm{i}}+T_{i}<t\right\}} \\
>x+l(x), N(t)=n
\end{array}\right) \\
& \geq \sum_{\mathrm{i}=1}^{n} P\left(\begin{array}{l}
\sum_{1 \leq \mathrm{k} \leq \mathrm{n}, \mathrm{k} \neq \mathrm{i}} X_{k} \mathrm{e}^{-\delta \omega_{\mathrm{k}}} 1_{\left\{\omega_{\mathrm{k}}<t\right\}} \\
+Z_{k} \mathrm{e}^{-\delta\left(\omega_{\mathrm{k}}+T_{k}\right)} 1_{\left\{\omega_{\mathrm{k}}+T_{k}<t\right\}}
\end{array}\right. \\
& >-l(x)),\left(X_{i} \mathrm{e}^{-\delta \omega_{\mathrm{i}}} 1_{\left\{\omega_{\mathrm{i}}<t\right\}}+Z_{i} \mathrm{e}^{-\delta\left(\omega_{i}+T_{i}\right)} 1_{\left\{\omega_{\mathrm{i}}+T_{i}<t\right\}}\right. \\
& >x+l(x), N(t)=n)
\end{aligned}
$$

If we apply the basic probability formula $P\left(\mathrm{~B} \cap \mathrm{A}^{\mathrm{c}}\right)=$ $P(\mathrm{~B})-P(\mathrm{~B} \cap \mathrm{A})$ and $P(\mathrm{AB})=P(\mathrm{~A} \mid \mathrm{B}) P(\mathrm{~B})$, we have:

$$
\begin{gathered}
\hat{J} \geq \sum_{i=1}^{n} P\left(\begin{array}{l}
X_{i} \mathrm{e}^{-\delta \omega_{\mathrm{i}}} 1_{\left\{\omega_{\mathrm{i}}<t\right\}}+Z_{i} \mathrm{e}^{-\delta\left(\omega_{i}+T_{i}\right)} 1_{\left\{\omega_{\mathrm{i}}+T_{i}<t\right\}} \\
>x+l(x), N(t)=n
\end{array}\right) \\
-\left(\begin{array}{l}
\sum_{i=1}^{n} P\left(\begin{array}{l}
\sum_{1 \leq \mathrm{k} \leq \mathrm{n}, \mathrm{k} \neq \mathrm{i}}\left(X_{k} \mathrm{e}^{-\delta \omega_{\mathrm{k}}} 1_{\left\{\omega_{\mathrm{k}}<t\right\}}\right. \\
+Z_{k} \mathrm{e}^{-\delta\left(\omega_{\mathrm{k}}+T_{k}\right)} 1_{\left\{\omega_{\mathrm{k}}+T_{k}<t\right\}} \leq-l(x)
\end{array}\right) \\
\left(\begin{array}{l}
X_{i} \mathrm{e}^{-\delta \omega_{\mathrm{i}}} 1_{\left\{\omega_{\mathrm{i}}<t\right\}}+Z_{i} \mathrm{e}^{-\delta\left(\omega_{i}+T_{i}\right)} 1_{\left\{\omega_{\mathrm{i}}+T_{i}<t\right\}} \\
>x+l(x), N(t)=n)
\end{array}\right)
\end{array}\right)
\end{gathered}
$$




$$
\begin{aligned}
& +\sum_{1 \leq \mathrm{k}<i \leq \mathrm{n},} P\left(X_{k} \mathrm{e}^{-\delta \omega_{\mathrm{k}}} 1_{\left\{\omega_{\mathrm{k}}<t\right\}}\right. \\
& +Z_{k} \mathrm{e}^{-\delta\left(\omega_{\mathrm{k}}+T_{k}\right)} 1_{\left\{\omega_{\mathrm{k}}+T_{k}<t\right\}}>x+l(\mathrm{x}) \mid X_{i} \mathrm{e}^{-\delta \omega_{\mathrm{i}}} 1_{\left\{\omega_{\mathrm{i}}<t\right\}} \\
& \left.\left.+Z_{i} \mathrm{e}^{-\delta\left(\omega_{i}+T_{i}\right)} 1_{\left\{\omega_{\mathrm{i}}+T_{i}<t\right\}}>x+l(x), N(t)=n\right)\right) \\
& P\left(X_{1}+Z_{1}>x+l(x)\right)=J_{1}(\mathrm{x}, l)-\left(J_{2}(\mathrm{x}, l)-J_{3}(\mathrm{x}, l)\right)
\end{aligned}
$$

Because of $H$ and $F$ belong to the class $\mathcal{L}$, the independent relationship between $X_{n}$ and $Z_{n}$ and the Lemma 3.5, we find Equation 4.6:

$$
\begin{aligned}
& \lim _{\mathrm{x} \rightarrow \infty} \inf _{\inf } \mathrm{t} \in \Lambda[0, \mathrm{~T}]_{\frac{J_{1}(x, l)}{\tilde{J}}}^{\tilde{J}} \\
& \geq \lim _{\mathrm{x} \rightarrow \infty} \inf _{\inf } \mathrm{t \in \Lambda [0,T]} \frac{J}{J^{*}} \geq 1
\end{aligned}
$$

Thus, we can conclude that the relation (3.19) can lead to the relation (4.7).

For $J_{2}(x, l)$, considering the independent relationship among $X_{k}, Z_{k}$ and $N(t)$ and the independent principle of probability $P\left(\mathrm{~A}_{1} \cup \mathrm{A}_{2} \mid \mathrm{B}\right) \leq P\left(\mathrm{~A}_{1} \mid \mathrm{B}\right)+P\left(\mathrm{~A}_{2} \mid \mathrm{B}\right)$, we derive:

$$
\begin{aligned}
& \left.J_{2}(\mathrm{x}, l) \leq \sum_{\mathrm{i}=1}^{n} \sum_{1 \leq \mathrm{k}<i \leq \mathrm{n},} P\left(\begin{array}{l}
X_{k} \mathrm{e}^{-\delta \omega_{\mathrm{k}}} 1_{\left\{\omega_{\mathrm{k}}<t\right\}}+Z_{k} \mathrm{e}^{-\delta\left(\omega_{\mathrm{k}}+T_{k}\right)} 1_{\left\{\omega_{\mathrm{k}}+T_{k}<t\right\}} \leq-\frac{l(\mathrm{x})}{\mathrm{n}}, \\
\left(X_{i} \mathrm{e}^{-\delta \omega_{\mathrm{i}}} 1_{\left\{\omega_{\mathrm{i}}<t\right\}}+Z_{i} \mathrm{e}^{-\delta\left(\omega_{i}+T_{i}\right)} 1_{\left\{\omega_{\mathrm{i}}+T_{i}<t\right\}}>x+l(x), N(t)=n\right)
\end{array}\right)\right) \\
& \leq \sum_{i=1}^{n} \sum_{1 \leq \mathrm{k}<i \leq \mathrm{n}}\left(P\left(X_{k} \mathrm{e}^{-\delta \omega_{\mathrm{k}}} 1_{\left\{\omega_{\mathrm{k}}<t\right\}} \leq-\frac{l(\mathrm{x})}{2 \mathrm{n}}\right),\left(X_{i} \mathrm{e}^{-\delta \omega_{\mathrm{i}}} 1_{\left\{\omega_{\mathrm{i}}<t\right\}}+Z_{i} \mathrm{e}^{-\delta\left(\omega_{i}+T_{i}\right)} 1_{\left\{\omega_{\mathrm{i}}+T_{i}<t\right\}}>x+l(x), N(t)=n\right)\right) \\
& \left.+P\left(Z_{k} \mathrm{e}^{-\delta\left(\omega_{\mathrm{k}}+T_{k}\right)} 1_{\left\{\omega_{\mathrm{k}}+T_{k}<t\right\}} \leq-\frac{l(\mathrm{x})}{2 \mathrm{n}}\right),\left(X_{i} \mathrm{e}^{-\delta \omega_{\mathrm{i}}} 1_{\left\{\omega_{\mathrm{i}}<t\right\}}+Z_{i} \mathrm{e}^{-\delta\left(\omega_{i}+T_{i}\right)} 1_{\left\{\omega_{\mathrm{i}}+T_{i}<t\right\}}>x+l(x), N(t)=n\right)\right) \\
& \leq \sum_{i=1}^{n} \sum_{1 \leq \mathrm{k}<i \leq \mathrm{n},}\left(P\left(X_{k} \mathrm{e}^{-\delta \omega_{\mathrm{k}}} 1_{\left\{\omega_{\mathrm{k}}<t\right\}} \leq-\frac{l(\mathrm{x})}{2 \mathrm{n}}, X_{i} \mathrm{e}^{-\delta \omega_{\mathrm{i}}} 1_{\left\{\omega_{\mathrm{i}}<t\right\}}>\frac{\mathrm{x}+l(\mathrm{x})}{2}, N(t)=n\right)+\right. \\
& +P\left(X_{k} \mathrm{e}^{-\delta \omega_{\mathrm{k}}} 1_{\left\{\omega_{\mathrm{k}}<t\right\}} \leq-\frac{l(\mathrm{x})}{2 \mathrm{n}}, Z_{i} \mathrm{e}^{-\delta\left(\omega_{i}+T_{i}\right)} 1_{\left\{\omega_{\mathrm{i}}+T_{i}<t\right\}}>\frac{\mathrm{x}+l(\mathrm{x})}{2}, N(t)=n\right) \\
& +P\left(Z_{k} \mathrm{e}^{-\delta\left(\omega_{\mathrm{k}}+T_{k}\right)} 1_{\left\{\omega_{\mathrm{k}}+T_{k}<t\right\}} \leq-\frac{l(\mathrm{x})}{2 \mathrm{n}}, X_{i} \mathrm{e}^{-\delta \omega_{\mathrm{i}}} 1_{\left\{\omega_{\mathrm{i}}<t\right\}}>\frac{\mathrm{x}+l(\mathrm{x})}{2}, N(t)=n\right) \\
& +P\left(Z_{k} \mathrm{e}^{-\delta\left(\omega_{\mathrm{k}}+T_{k}\right)} 1_{\left\{\omega_{\mathrm{k}}+T_{k}<t\right\}} \leq-\frac{l(\mathrm{x})}{2 \mathrm{n}}, Z_{i} \mathrm{e}^{-\delta\left(\omega_{i}+T_{i}\right)} 1_{\left\{\omega_{\mathrm{i}}+T_{i}<t\right\}}>\frac{\mathrm{x}+l(\mathrm{x})}{2}, N(t)=n\right) \\
& \leq \sum_{i=1}^{n} \sum_{1 \leq \mathrm{k}<i \leq \mathrm{n},}\left(P\left(\left|X_{k} \mathrm{e}^{-\delta \omega_{\mathrm{k}}} 1_{\left\{\omega_{\mathrm{k}}<t\right\}}\right| \geq \frac{l(\mathrm{x})}{2 \mathrm{n}}, X_{i} \mathrm{e}^{-\delta \omega_{\mathrm{i}}} 1_{\left\{\omega_{\mathrm{i}}<t\right\}}>\frac{\mathrm{x}+l(\mathrm{x})}{2}, N(t)=n\right)\right. \\
& +P\left(\left|X_{k} \mathrm{e}^{-\delta \omega_{\mathrm{k}}} 1_{\left\{\omega_{\mathrm{k}}<t\right\}}\right| \geq \frac{l(\mathrm{x})}{2 \mathrm{n}}, Z_{i} \mathrm{e}^{-\delta\left(\omega_{i}+T_{i}\right)} 1_{\left\{\omega_{\mathrm{i}}+T_{i}<t\right\}}>\frac{\mathrm{x}+l(\mathrm{x})}{2}, N(t)=n\right) \\
& +P\left(\left|Z_{k} \mathrm{e}^{-\delta\left(\omega_{\mathrm{k}}+T_{k}\right)} 1_{\left\{\omega_{\mathrm{k}}+T_{k}<t\right\}}\right| \geq \frac{l(\mathrm{x})}{2 \mathrm{n}}, X_{i} \mathrm{e}^{-\delta \omega_{\mathrm{i}}} 1_{\left\{\omega_{\mathrm{i}}<t\right\}}>\frac{\mathrm{x}+l(\mathrm{x})}{2}, N(t)=n\right) \\
& +P\left(\left|Z_{k} \mathrm{e}^{-\delta\left(\omega_{\mathrm{k}}+T_{k}\right)} 1_{\left\{\omega_{\mathrm{k}}+T_{k}<t\right\}}\right| \geq \frac{l(\mathrm{x})}{2 \mathrm{n}}, Z_{i} \mathrm{e}^{-\delta\left(\omega_{i}+T_{i}\right)} 1_{\left\{\omega_{\mathrm{i}}+T_{i}<t\right\}}>\frac{\mathrm{x}+l(\mathrm{x})}{2}, N(t)=n\right)
\end{aligned}
$$

For the last four term of relation (4.8), we can apply the formula $P(\mathrm{AB})=P(\mathrm{~A} \mid \mathrm{B}) P(\mathrm{~B})$ and the definition of pSQAI respectively:

$$
\begin{aligned}
& \lim _{\mathrm{x} \rightarrow \infty} \sup _{\mathrm{t} \in \Lambda[0, \mathrm{~T}]} P\left(\left|X_{k} \mathrm{e}^{-\delta \omega_{\mathrm{k}}} 1_{\left\{\omega_{\mathrm{k}}<t\right\}}\right| \geq \frac{l(\mathrm{x})}{2 \mathrm{n}} \mid X_{i} \mathrm{e}^{-\delta \omega_{\mathrm{i}}} 1_{\left\{\omega_{\mathrm{i}}<t\right\}}>\frac{\mathrm{x}+l(\mathrm{x})}{2}\right) \times \mathrm{P}\left(X_{i} \mathrm{e}^{-\delta \omega_{\mathrm{i}}} 1_{\left\{\omega_{\mathrm{i}}<t\right\}}>\frac{\mathrm{x}+l(\mathrm{x})}{2}\right)=0 \\
& \lim _{\mathrm{x} \rightarrow \infty} \sup \sup _{\mathrm{t} \in \Lambda[0, \mathrm{~T}]} P\left(\left|X_{k} \mathrm{e}^{-\delta \omega_{\mathrm{k}}} 1_{\left\{\omega_{\mathrm{k}}<t\right\}}\right| \geq \frac{l(\mathrm{x})}{2 \mathrm{n}} \mid Z_{i} \mathrm{e}^{-\delta\left(\omega_{i}+T_{i}\right)} 1_{\left\{\omega_{\mathrm{i}}+T_{i}<t\right\}}>\frac{\mathrm{x}+l(\mathrm{x})}{2}\right) \times \mathrm{P}\left(Z_{i} \mathrm{e}^{-\delta\left(\omega_{i}+T_{i}\right)} 1_{\left\{\omega_{\mathrm{i}}+T_{i}<t\right\}}>\frac{\mathrm{x}+l(\mathrm{x})}{2}\right)=0
\end{aligned}
$$




$$
\begin{aligned}
& \lim _{\mathrm{x} \rightarrow \infty} \sup \sup _{\mathrm{t} \in \Lambda[0, \mathrm{~T}]} P\left(\left|Z_{k} \mathrm{e}^{-\delta\left(\omega_{\mathrm{k}}+T_{k}\right)} 1_{\left\{\omega_{\mathrm{k}}+T_{k}<t\right\}}\right| \geq \frac{l(\mathrm{x})}{2 \mathrm{n}} \mid X_{i} \mathrm{e}^{-\delta \omega_{\mathrm{i}}} 1_{\left\{\omega_{\mathrm{i}}<t\right\}}>\frac{\mathrm{x}+l(\mathrm{x})}{2}\right) \times \mathrm{P}\left(X_{i} \mathrm{e}^{-\delta \omega_{\mathrm{i}}} 1_{\left\{\omega_{\mathrm{i}}<t\right\}}>\frac{\mathrm{x}+l(\mathrm{x})}{2}\right)=0 \\
& \lim _{\mathrm{x} \rightarrow \infty} \sup \sup _{\mathrm{t} \in \Lambda[0, \mathrm{~T}]} P\left(\left|Z_{k} \mathrm{e}^{-\delta\left(\omega_{\mathrm{k}}+T_{k}\right)} 1_{\left\{\omega_{\mathrm{k}}+T_{k}<t\right\}}\right| \geq \frac{l(\mathrm{x})}{2 \mathrm{n}} \mid Z_{i} \mathrm{e}^{-\delta\left(\omega_{i}+T_{i}\right)} 1_{\left\{\omega_{\mathrm{i}}+T_{i}<t\right\}}>\frac{\mathrm{x}+l(\mathrm{x})}{2}\right) \\
& \times \mathrm{P}\left(Z_{i} \mathrm{e}^{-\delta\left(\omega_{i}+T_{i}\right)} 1_{\left\{\omega_{\mathrm{i}}+T_{i}<t\right\}}>\frac{\mathrm{x}+l(\mathrm{x})}{2}\right)=0
\end{aligned}
$$

Finally, $J_{2}(x, l)$ also goes to zero.

And then, we turn to $J_{3}(x, l)$.

$$
\begin{aligned}
& J_{3}(\mathrm{x}, l) \leq \sum_{1 \leq \mathrm{k}<i \leq \mathrm{n}}\left(P\left(X_{k} \mathrm{e}^{-\delta \omega_{\mathrm{k}}} 1_{\left\{\omega_{\mathrm{k}}<t\right\}} \geq \mathrm{x}+l(\mathrm{x}) \mid X_{i} \mathrm{e}^{-\delta \omega_{\mathrm{i}}} 1_{\left\{\omega_{\mathrm{i}}<t\right\}}+Z_{i} \mathrm{e}^{-\delta\left(\omega_{i}+T_{i}\right)} 1_{\left\{\omega_{\mathrm{i}}+T_{i}<t\right\}}>x+l(x), N(t)=n\right)\right) \\
& +P\left(\begin{array}{l}
\left.Z_{k} \mathrm{e}^{-\delta\left(\omega_{\mathrm{k}}+T_{k}\right)} 1_{\left\{\omega_{\mathrm{k}}+T_{k}<t\right\}} \geq \mathrm{x}+l(\mathrm{x}) \mid X_{i} \mathrm{e}^{-\delta \omega_{\mathrm{i}}} 1_{\left\{\omega_{\mathrm{i}}<t\right\}}\right) \\
+Z_{i} \mathrm{e}^{-\delta\left(\omega_{i}+T_{i}\right)} 1_{\left\{\omega_{\mathrm{i}}+T_{i}<t\right\}}>x+l(x), N(t)=n
\end{array}\right) P\left(X_{1}+Z_{1}>x+l(x)\right. \\
& \leq \sum_{1 \leq \mathrm{k}<i \leq \mathrm{n}}\left(P\left(X_{k} \mathrm{e}^{-\delta \omega_{\mathrm{k}}} 1_{\left\{\omega_{\mathrm{k}}<t\right\}} \geq \mathrm{x}+l(\mathrm{x}) \mid X_{i} \mathrm{e}^{-\delta \omega_{\mathrm{i}}} 1_{\left\{\omega_{\mathrm{i}}<t\right\}}>x+l(x), N(t)=n\right)\right. \\
& +P\left(X_{k} \mathrm{e}^{-\delta \omega_{\mathrm{k}}} 1_{\left\{\omega_{\mathrm{k}}<t\right\}} \geq \mathrm{x}+l(\mathrm{x}) \mid Z_{i} \mathrm{e}^{-\delta\left(\omega_{i}+T_{i}\right)} 1_{\left\{\omega_{\mathrm{i}}+T_{i}<t\right\}}>x+l(x), N(t)=n\right) \\
& +P\left(Z_{k} \mathrm{e}^{-\delta\left(\omega_{\mathrm{k}}+T_{k}\right)} 1_{\left\{\omega_{\mathrm{k}}+T_{k}<t\right\}} \geq \mathrm{x}+l(\mathrm{x}) \mid X_{i} \mathrm{e}^{-\delta \omega_{\mathrm{i}}} 1_{\left\{\omega_{\mathrm{i}}<t\right\}}>x+l(x), N(t)=n\right) \\
& \left.+P\left(Z_{k} \mathrm{e}^{-\delta\left(\omega_{\mathrm{k}}+T_{k}\right)} 1_{\left\{\omega_{\mathrm{k}}+T_{k}<t\right\}} \geq \mathrm{x}+l(\mathrm{x}) \mid Z_{i} \mathrm{e}^{-\delta\left(\omega_{i}+T_{i}\right)} 1_{\left\{\omega_{\mathrm{i}}+T_{i}<t\right\}}>x+l(x), N(t)=n\right)\right)
\end{aligned}
$$

We try to apply the definition of pSQAI and the condition of $H \in \mathcal{L}$ and $F \in \mathcal{L}$ again:

$$
\begin{aligned}
& \lim _{\mathrm{x} \rightarrow \infty} \sup \sup _{\mathrm{t} \in \Lambda[0, \mathrm{~T}]} P\left(X_{k} \mathrm{e}^{-\delta \omega_{\mathrm{k}}} 1_{\left\{\omega_{\mathrm{k}}<t\right\}} \geq \mathrm{x}+l(\mathrm{x}) \mid X_{i} \mathrm{e}^{-\delta \omega_{\mathrm{i}}} 1_{\left\{\omega_{\mathrm{i}}<t\right\}}>\mathrm{x}+l(\mathrm{x})\right)=0 \\
& \lim _{\mathrm{x} \rightarrow \infty} \sup \sup _{\mathrm{t} \in \Lambda[0, \mathrm{~T}]} P\left(X_{\mathrm{k}} \mathrm{e}^{-\delta \omega_{\mathrm{k}}} 1_{\left\{\omega_{\mathrm{k}}<t\right\}} \geq \mathrm{x}+l(\mathrm{x}) \mid Z_{i} \mathrm{e}^{-\delta\left(\omega_{i}+T_{i}\right)} 1_{\left\{\omega_{\mathrm{i}}+T_{i}<t\right\}}>\mathrm{x}+l(\mathrm{x})\right)=0 \\
& \lim _{\mathrm{x} \rightarrow \infty} \sup \sup _{\mathrm{t} \in \Lambda[0, \mathrm{~T}]} P\left(Z_{k} \mathrm{e}^{-\delta\left(\omega_{\mathrm{k}}+T_{k}\right)} 1_{\left\{\omega_{\mathrm{k}}+T_{k}<t\right\}} \geq \mathrm{x}+l(\mathrm{x}) \mid X_{i} \mathrm{e}^{-\delta \omega_{\mathrm{i}}} 1_{\left\{\omega_{\mathrm{i}}<t\right\}}>\mathrm{x}+l(\mathrm{x})\right)=0 \\
& \lim _{\mathrm{x} \rightarrow \infty} \sup \sup _{\mathrm{t} \in \Lambda[0, \mathrm{~T}]} P\left(Z_{k} \mathrm{e}^{-\delta\left(\omega_{\mathrm{k}}+T_{k}\right)} 1_{\left\{\omega_{\mathrm{k}}+T_{k}<t\right\}} \geq \mathrm{x}+l(\mathrm{x}) \mid Z_{i} \mathrm{e}^{-\delta\left(\omega_{i}+T_{i}\right)} 1_{\left\{\omega_{\mathrm{i}}+T_{i}<t\right\}}>\mathrm{x}+l(\mathrm{x})\right)=0
\end{aligned}
$$

Consequently, $J_{3}(x, l)$ also goes to zero.

Thus, a combination of $J_{1}(x, l), J_{2}(x, l)$ and $J_{3}(x, l)$ can hold the relation (4.4).

In the context of the above proof, we can continue our proof of relation (4.3).

If we consider the following notation in relation (4.3). we define:

$$
\begin{aligned}
& A=\left\{\sum_{k=1}^{n}\left(X_{k} \mathrm{e}^{-\delta \omega_{\mathrm{k}}} 1_{\left\{\omega_{\mathrm{k}}<t\right\}}+Z_{k} \mathrm{e}^{-\delta\left(\omega_{\mathrm{k}}+T_{k}\right)} 1_{\left\{\omega_{\mathrm{k}}+T_{k}<t\right\}}>x\right\}\right. \\
& B=\left\{\sum_{j=1}^{n}\left(X_{j} \mathrm{e}^{-\delta \omega_{\mathrm{j}}} 1_{\left\{\omega_{\mathrm{j}}<t\right\}}+Z_{j} \mathrm{e}^{-\delta\left(\omega_{\mathrm{j}}+T_{j}\right)} 1_{\left\{\omega_{\mathrm{j}}+T_{j}<t\right\}}>x-l(x)\right\}\right.
\end{aligned}
$$

We can take the same logic reasoning of the general case of the probability that $P(\mathrm{~A})=P(\mathrm{AB})+P\left(\mathrm{AB}^{\mathrm{c}}\right)$ to the following relation, we find Equation 4.7: 


$$
\begin{aligned}
& \hat{J}=P\left(\sum _ { k = 1 } ^ { n } \left(X_{k} \mathrm{e}^{-\delta \omega_{\mathrm{k}}} 1_{\left\{\omega_{\mathrm{k}}<t\right\}}+Z_{k} \mathrm{e}^{-\delta\left(\omega_{\mathrm{k}}+T_{k}\right)} 1_{\left\{\omega_{\mathrm{k}}+T_{k}<t\right\}}>x, N(t)=n\right.\right. \\
& \left.\cup_{j=1}^{n} X_{j} \mathrm{e}^{-\delta \omega_{\mathrm{j}}} 1_{\left\{\omega_{\mathrm{j}}<t\right\}}+Z_{j} \mathrm{e}^{-\delta\left(\omega_{\mathrm{j}}+T_{j}\right)} 1_{\left\{\omega_{\mathrm{j}}+T_{j}<t\right\}}>x-l(x), N(t)=n\right) \\
& +P\left(\sum _ { k = 1 } ^ { n } \left(X_{k} \mathrm{e}^{-\delta \omega_{\mathrm{k}}} 1_{\left\{\omega_{\mathrm{k}}<t\right\}}+Z_{k} \mathrm{e}^{-\delta\left(\omega_{\mathrm{k}}+T_{k}\right)} 1_{\left\{\omega_{\mathrm{k}}+T_{k}<t\right\}}>x, N(t)=n\right.\right. \\
& \left.\bigcup_{j=1}^{n} X_{j} \mathrm{e}^{-\delta \omega_{\mathrm{j}}} 1_{\left\{\omega_{\mathrm{j}}<t\right\}}+Z_{j} \mathrm{e}^{-\delta\left(\omega_{\mathrm{j}}+T_{j}\right)} 1_{\left\{\omega_{\mathrm{j}}+T_{j}<t\right\}} \leq x-l(x), N(t)=n\right)
\end{aligned}
$$

After applying the $P\left(\bigcup_{k=1}^{n} B\right) \leq \sum_{k=1}^{n} P(B) \leq \sum_{k=1}^{n}(A B)$ to relation (4.9), Therefore, we obtain:

$$
\begin{aligned}
& \hat{J} \leq \sum_{k=1}^{n} P\left(X_{j} \mathrm{e}^{-\delta \omega_{\mathrm{j}}} 1_{\left\{\omega_{\mathrm{j}}<t\right\}}+Z_{j} \mathrm{e}^{-\delta\left(\omega_{\mathrm{j}}+T_{j}\right)} 1_{\left\{\omega_{\mathrm{j}}+T_{j}<t\right\}}>x-l(x), N(t)=n\right) \\
& +P\left(\sum_{k=1}^{n}\left(X_{k} \mathrm{e}^{-\delta \omega_{\mathrm{k}}} 1_{\left\{\omega_{\mathrm{k}}<t\right\}}+Z_{k} \mathrm{e}^{-\delta\left(\omega_{\mathrm{k}}+T_{k}\right)} 1_{\left\{\omega_{\mathrm{k}}+T_{k}<t\right\}}>x, N(t)=n\right), \frac{x}{n}<X_{j}+Z_{j} \leq x-l(x)\right)
\end{aligned}
$$

After the transformation of the inequality from:

$\left.\frac{x}{n}<X_{j}+Z_{j} \leq x-l(x)\right)$ to $\left.-\frac{x}{n}<-\left(X_{j}+Z_{j}\right) \leq l(x)-x\right)$

And adding to the set of :

$$
\left\{\sum_{k=1}^{n}\left(X_{k} \mathrm{e}^{-\delta \omega_{\mathrm{k}}} 1_{\left\{\omega_{\mathrm{k}}<t\right\}}+Z_{k} \mathrm{e}^{-\delta\left(\omega_{\mathrm{k}}+T_{k}\right)} 1_{\left\{\omega_{\mathrm{k}}+T_{k}<t\right\}}>x\right\}\right.
$$

We derive:

$$
\begin{aligned}
& \hat{J} \leq \sum_{k=1}^{n} P\left(\begin{array}{l}
\left.X_{k} \mathrm{e}^{-\delta \omega_{\mathrm{k}}} 1_{\left\{\omega_{\mathrm{k}}<t\right\}}+Z_{k} \mathrm{e}^{-\delta\left(\omega_{\mathrm{k}}+T_{k}\right)} 1_{\left\{\omega_{\mathrm{k}}+T_{k}<t\right\}}\right), N(t)=n \\
>x-l(x), N(t)
\end{array}\right) \\
& +\sum_{j=1}^{n} P\left(\begin{array}{l}
X_{k} \mathrm{e}^{-\delta \omega_{\mathrm{k}}} 1_{\left\{\omega_{\mathrm{k}}<t\right\}} \\
+Z_{k} \mathrm{e}^{-\delta\left(\omega_{\mathrm{k}}+T_{k}\right)} 1_{\left\{\omega_{\mathrm{k}}+T_{k}<t\right\}} \\
<l(\mathrm{x}), N(\mathrm{t})=\mathrm{n}
\end{array}\right), \\
& \left.X_{j} \mathrm{e}^{-\delta \omega_{\mathrm{j}}} 1_{\left\{\omega_{\mathrm{j}}<t\right\}}+Z_{j} \mathrm{e}^{-\delta\left(\omega_{\mathrm{j}}+T_{j}\right)} 1_{\left\{\omega_{\mathrm{j}}+T_{j}<t\right\}}>\frac{x}{n}, N(t)=n\right)
\end{aligned}
$$

Because of the face that:

$$
\begin{aligned}
& \left\{X_{k} \mathrm{e}^{-\delta \omega_{\mathrm{k}}} 1_{\left\{\omega_{\mathrm{k}}<t\right\}}+Z_{k} \mathrm{e}^{-\delta\left(\omega_{\mathrm{k}}+T_{k}\right)} 1_{\left\{\omega_{\mathrm{k}}+T_{k}<t\right\}}>x-l(x)\right\} \\
& \subseteq\left\{X_{k} \mathrm{e}^{-\delta \omega_{\mathrm{k}}} 1_{\left\{\omega_{\mathrm{k}}<t\right\}}+Z_{k}>x-l(x)\right\}
\end{aligned}
$$

Therefore:

$$
\begin{aligned}
& P\left(\begin{array}{l}
X_{k} \mathrm{e}^{-\delta \omega_{\mathrm{k}}} 1_{\left\{\omega_{\mathrm{k}}<t\right\}}+Z_{k} \mathrm{e}^{-\delta\left(\omega_{\mathrm{k}}+T_{k}\right)} 1_{\left\{\omega_{\mathrm{k}}+T_{k}<t\right\}} \\
>x-l(x), N(t)=n
\end{array}\right) \\
& \leq P\left(X_{k} \mathrm{e}^{-\delta \omega_{\mathrm{k}}} 1_{\left\{\omega_{\mathrm{k}}<t\right\}}+Z_{k}>x-l(x)\right.
\end{aligned}
$$

In addition, we all have known that the Boole's inequality can lead to the conclusion:

$$
\begin{aligned}
& P\left(\begin{array}{l}
\bigcap_{1 \leq k \leq n, k \neq j} X_{k} \mathrm{e}^{-\delta \omega_{\mathrm{k}}} 1_{\left\{\omega_{\mathrm{k}}<t\right\}} \\
+Z_{k} \mathrm{e}^{-\delta\left(\omega_{\mathrm{k}}+T_{k}\right)} 1_{\left\{\omega_{\mathrm{k}}+T_{k}<t\right\}}<\frac{l(x)}{k-1}, N(t)=n
\end{array}\right) \\
& \leq P\left(\begin{array}{l}
\sum_{k} \mathrm{e}^{-\delta \omega_{\mathrm{k}}} 1_{\left\{\omega_{\mathrm{k}}<t\right\}} \\
+Z_{k} \mathrm{e}^{-\delta\left(\omega_{\mathrm{k}}+T_{k}\right)} 1_{\left\{\omega_{\mathrm{k}}+T_{k}<t\right\}} \\
<l(\mathrm{x}), N(\mathrm{t})=\mathrm{n}
\end{array}\right)
\end{aligned}
$$

Then we apply the simple logic reasoning and De Morgan rule, we may find:

$$
\begin{aligned}
& P\left(\sum_{1 \leq k \leq n, k \neq j}\left(\begin{array}{l}
X_{k} \mathrm{e}^{-\delta \omega_{\mathrm{k}}} 1_{\left\{\omega_{\mathrm{k}}<t\right\}} \\
+Z_{k} \mathrm{e}^{-\delta\left(\omega_{\mathrm{k}}+T_{k}\right)} 1_{\left\{\omega_{\mathrm{k}}+T_{k}<t\right\}} \\
>l(\mathrm{x}), N(\mathrm{t})=\mathrm{n}
\end{array}\right)\right) \\
& \leq P\left(\begin{array}{l}
\bigcup_{1 \leq k \leq n, k \neq j} X_{k} \mathrm{e}^{-\delta \omega_{\mathrm{k}}} 1_{\left\{\omega_{\mathrm{k}}<t\right\}} \\
+Z_{k} \mathrm{e}^{-\delta\left(\omega_{\mathrm{k}}+T_{k}\right)} 1_{\left\{\omega_{\mathrm{k}}+T_{k}<t\right\}}>\frac{l(x)}{k-1}, N(t)=n
\end{array}\right)
\end{aligned}
$$

Hence, we get: 


$$
\hat{j} \leq \sum_{k=1}^{n} P\left(X_{k} \mathrm{e}^{-\delta \omega_{\mathrm{k}}} 1_{\left\{\omega_{\mathrm{k}}<t\right\}}+Z_{k}>x-l(x)\right)+\sum_{k=1}^{n} P\left(\begin{array}{l}
U_{1 \leq k \leq n, k \neq j} X_{k} \mathrm{e}^{-\delta \omega_{\mathrm{k}}} 1_{\left\{\omega_{\mathrm{k}}<t\right\}}+Z_{k} \mathrm{e}^{-\delta\left(\omega_{\mathrm{k}}+T_{k}\right)} 1_{\left\{\omega_{\mathrm{k}}+T_{k}<t\right\}}>\frac{l(x)}{k-1}, \\
X_{j} \mathrm{e}^{-\delta \omega_{\mathrm{j}}} 1_{\left\{\omega_{\mathrm{j}}<t\right\}}+Z_{j} \mathrm{e}^{-\delta\left(\omega_{\mathrm{j}}+T_{j}\right)} 1_{\left\{\omega_{\mathrm{j}}+T_{j}<t\right\}}>\frac{x}{n}, N(t)=n
\end{array}\right)
$$

According to the Boole's inequality:

$$
\begin{aligned}
& P\left(\bigcup_{1 \leq k \leq n, k \neq j} X_{k} \mathrm{e}^{-\delta \omega_{k}} 1_{\left\{\omega_{k}<t\right\}}+Z_{k} \mathrm{e}^{-\delta\left(\omega_{k}+T_{k}\right)} 1_{\left\{\omega_{\mathrm{k}}+T_{k}<t\right\}}>\frac{l(x)}{k-1}, N(t)=n\right) \\
& \leq \sum_{1 \leq k \leq n, k \neq j} P\left(X_{k} \mathrm{e}^{-\delta \omega_{\mathrm{k}}} 1_{\left\{\omega_{\mathrm{k}}<t\right\}}+Z_{k} \mathrm{e}^{-\delta\left(\omega_{\mathrm{k}}+T_{k}\right)} 1_{\left\{\omega_{\mathrm{k}}+T_{k}<t\right\}}>\frac{l(x)}{k-1}, N(\mathrm{t})=\mathrm{n}\right)
\end{aligned}
$$

Then, we have:

$$
\begin{aligned}
& \hat{J} \leq \sum_{k=1}^{n} P\left(X_{k} \mathrm{e}^{-\delta \omega_{\mathrm{k}}} 1_{\left\{\omega_{\mathrm{k}}<t\right\}}+Z_{k} \mathrm{e}^{-\delta\left(\omega_{\mathrm{k}}+T_{k}\right)} 1_{\left\{\omega_{\mathrm{k}}+T_{k}<t\right\}}>x-l(x)\right) \\
& +\sum_{j=1}^{n} \sum_{1 \leq k \leq n, j \neq k}^{n}\left(\begin{array}{l}
P\left(X_{k} \mathrm{e}^{-\delta \omega_{\mathrm{k}}} 1_{\left\{\omega_{\mathrm{k}}<t\right\}}+Z_{k} \mathrm{e}^{-\delta\left(\omega_{\mathrm{k}}+T_{k}\right)} 1_{\left\{\omega_{\mathrm{k}}+T_{k}<t\right\}}>\frac{l(x)}{k-1}, N(t)=n\right) \\
X_{j} \mathrm{e}^{-\delta \omega_{\mathrm{j}}} 1_{\left\{\omega_{\mathrm{j}}<t\right\}}+Z_{j} \mathrm{e}^{-\delta\left(\omega_{\mathrm{j}}+T_{j}\right)} 1_{\left\{\omega_{\mathrm{j}}+T_{j}<t\right\}}>\frac{x}{n}, N(t)=n
\end{array}\right) \\
& \leq \sum_{k=1}^{n} P\left(X_{k} \mathrm{e}^{-\delta \omega_{\mathrm{k}}} 1_{\left\{\omega_{\mathrm{k}}<t\right\}}+Z_{k} \mathrm{e}^{-\delta\left(\omega_{\mathrm{k}}+T_{k}\right)} 1_{\left\{\omega_{\mathrm{k}}+T_{k}<t\right\}}>x-l(x)\right) \\
& +\sum_{k=1}^{n} \sum_{1 \leq k \leq n, j \neq k}^{n}\left(\begin{array}{l}
P\left(X_{k} \mathrm{e}^{-\delta \omega_{\mathrm{k}}} 1_{\left\{\omega_{\mathrm{k}}<t\right\}}>\frac{l(x)}{k-1} \mid X_{j} \mathrm{e}^{-\delta \omega_{\mathrm{j}}} 1_{\left\{\omega_{\mathrm{j}}<t\right\}}+Z_{j} \mathrm{e}^{-\delta\left(\omega_{\mathrm{j}}+T_{j}\right)} 1_{\left\{\omega_{\mathrm{j}}+T_{j}<t\right\}}>\frac{x}{n}, N(t)=n\right) \\
\left.+\left(Z_{k} \mathrm{e}^{-\delta\left(\omega_{\mathrm{k}}+T_{k}\right)} 1_{\left\{\omega_{\mathrm{k}}+T_{k}<t\right\}}>\frac{l(x)}{k-1} \mid X_{j} \mathrm{e}^{-\delta \omega_{\mathrm{j}}} 1_{\left\{\omega_{\mathrm{j}}<t\right\}}+Z_{j} \mathrm{e}^{-\delta\left(\omega_{\mathrm{j}}+T_{j}\right)} 1_{\left\{\omega_{\mathrm{j}}+T_{j}<t\right\}}>\frac{x}{n}, N(t)=n\right)\right) \\
\leq \sum_{k=1}^{n} P\left(X_{k} \mathrm{e}^{-\delta \omega_{\mathrm{k}}} 1_{\left\{\omega_{\mathrm{k}}<t\right\}}+Z_{k}>x-l(x)\right) \\
+\sum_{j=1}^{n} \sum_{1 \leq k \leq n, j \neq k}\left(\begin{array}{l}
\left.+X_{k} \mathrm{e}^{-\delta \omega_{\mathrm{k}}} 1_{\left\{\omega_{\mathrm{k}}<t\right\}}>\frac{l(x)}{k-1} \mid X_{j} \mathrm{e}^{-\delta \omega_{\mathrm{j}}} 1_{\left\{\omega_{\mathrm{j}}<t\right\}}>\frac{x}{n}, N(t)=n\right) \\
+P\left(X_{k} \mathrm{e}^{-\delta \omega_{\mathrm{k}}} 1_{\left\{\omega_{\mathrm{k}}<t\right\}}>\frac{l(x)}{k-1} \mid Z_{j} \mathrm{e}^{-\delta\left(\omega_{\mathrm{j}}+T_{j}\right)} 1_{\left\{\omega_{\mathrm{j}}+T_{j}<t\right\}}>\frac{x}{n}, N(t)=n\right) \\
+P\left(Z_{k} \mathrm{e}^{-\delta\left(\omega_{\mathrm{k}}+T_{k}\right)} 1_{\left\{\omega_{\mathrm{k}}+T_{k}<t\right\}}>\frac{l(x)}{k-1} \mid X_{j} \mathrm{e}^{-\delta \omega_{\mathrm{j}}} 1_{\left\{\omega_{\mathrm{j}}<t\right\}}>\frac{x}{n}, N(t)=n\right)
\end{array}\right) \\
+P\left(Z_{k} \mathrm{e}^{-\delta\left(\omega_{\mathrm{k}}+T_{k}\right)} 1_{\left\{\omega_{\mathrm{k}}+T_{k}<t\right\}}>\frac{l(x)}{k-1} \mid Z_{j} \mathrm{e}^{-\delta\left(\omega_{\mathrm{j}}+T_{j}\right)} 1_{\left\{\omega_{\mathrm{j}}+T_{j}<t\right\}}>\frac{x}{n}, N(t)=n\right)=J_{4}(\mathrm{x}, l)+J_{5}(\mathrm{x}, l)
\end{array}\right.
\end{aligned}
$$

By the $H$ and $F$ belonging to the class $\mathcal{L}$, we should consider Lemma 3.6, for every $1 \leq k \leq n$ :

$$
\begin{aligned}
& \limsup _{\mathrm{x} \rightarrow \infty} \sup _{\mathrm{t} \in \Lambda[0, \mathrm{~T}]} \frac{J_{4}(\mathrm{x}, l)}{\tilde{J}} \leq \limsup _{\mathrm{x} \rightarrow \infty} \sup _{\mathrm{t} \in \Lambda[0, \mathrm{~T}]} \frac{\sum_{k=1}^{n} P\left(X_{k} \mathrm{e}^{-\delta \omega_{\mathrm{k}}} 1_{\left\{\omega_{\mathrm{k}}<t\right\}}+Z_{k}>x-l(x)\right)}{\tilde{J}} \\
& \limsup _{\mathrm{x} \rightarrow \infty} \sup _{\mathrm{t} \in \Lambda[0, \mathrm{~T}]} \frac{\hat{J}}{\tilde{J}} \leq 1
\end{aligned}
$$


For $J_{5}$, if the $H$ and $F$ belong to the class $\mathcal{L}$ and the property of pSQAI dependent structure, we derive that:

$$
\begin{aligned}
& \limsup _{\mathrm{x} \rightarrow \infty} P\left(X_{k} \mathrm{e}^{-\delta \omega_{\mathrm{k}}} 1_{\left\{\omega_{\mathrm{k}}<t\right\}}>\frac{l(x)}{\mathrm{k}-1} \mid X_{j} \mathrm{e}^{-\delta \omega_{j}} 1_{\left\{\omega_{j}<t\right\}}>\frac{\mathrm{x}}{\mathrm{n}}, N(\mathrm{t})=\mathrm{n}\right)=0 \\
& \limsup _{\mathrm{x} \rightarrow \infty} P\left(X_{k} \mathrm{e}^{-\delta \omega_{\mathrm{k}}} 1_{\left\{\omega_{\mathrm{k}}<t\right\}}>\frac{l(x)}{\mathrm{k}-1} \mid Z_{j} \mathrm{e}^{-\delta\left(\omega_{j}+T_{j}\right)} 1_{\left\{\omega_{j}+T_{j}<t\right\}}>\frac{\mathrm{x}}{\mathrm{n}}, N(\mathrm{t})=\mathrm{n}\right)=0 \\
& \limsup _{\mathrm{x} \rightarrow \infty} P\left(Z_{k} \mathrm{e}^{-\delta\left(\omega_{k}+T_{k}\right)} 1_{\left\{\omega_{k}+T_{k}<t\right\}}>\frac{\mathrm{x}}{\mathrm{n}}>\frac{l(x)}{\mathrm{k}-1} \mid X_{j} \mathrm{e}^{-\delta \omega_{j}} 1_{\left\{\omega_{j}<t\right\}}>\frac{\mathrm{x}}{\mathrm{n}}, N(\mathrm{t})=\mathrm{n}\right)=0 \\
& \limsup _{\mathrm{x} \rightarrow \infty} P\left(Z_{k} \mathrm{e}^{-\delta\left(\omega_{k}+T_{k}\right)} 1_{\left\{\omega_{k}+T_{k}<t\right\}}>\frac{\mathrm{x}}{\mathrm{n}}>\frac{l(x)}{\mathrm{k}-1} \mid Z_{j} \mathrm{e}^{-\delta\left(\omega_{j}+T_{j}\right)} 1_{\left\{\omega_{j}+T_{j}<t\right\}}>\frac{\mathrm{x}}{\mathrm{n}}, N(\mathrm{t})=\mathrm{n}\right)
\end{aligned}
$$

Thus, combining the $J_{4}(x, l)$ and $J_{5}(x, l)$, we hold the relation (4.3).

The following section will discuss the asymptotic behavior of $P\left(R_{\delta}^{*}(t, x)>x\right)$ in detail. Because we are not only interested in the asymptotic behavior of $P\left(R_{\delta}^{*}(t, x)>x\right)$ involved the independent structure of the claim size and the arrival times of successive claims, but also in the dependent structure in the renewal risk model.

It ends the proof of Lemma (4.1).

\section{Lemma 4.2}

Assume that the $\left\{X_{n}, Y_{n} ; n \geq 1\right\},\left\{\omega_{n} ; n \geq 1\right\}$ and $\left\{T_{n}\right.$; $n \geq 1\}$ are mutually independent. Consider the renewal risk model, we also assume that $X_{n}$ and $Y_{n}$ not necessarily independent. Let claim size $\left\{X_{n} ; n \geq 1\right\}$ and by-claim parts $\left\{Z_{n} ; n \geq 1\right\}$ follows pSQAI dependent structure with common distribution functions $H \in \mathcal{L} \cap \mathcal{D}$ and $F \in \mathcal{L} \cap \mathcal{D}$. The inter-arrival time $\left\{Y_{n} ; n \geq 1\right\}$ with common distribution $V$ are WLOD and also suppose the relation (2.11) and (2.12) hold. Then for any fixed $T \in \Lambda$ Equation 4.8:

$$
\begin{aligned}
& P\left(R_{\delta}^{*}(t, x)>x\right) \sim \int_{0}^{t}\left(\bar{H}\left(\mathrm{x} e^{\delta t^{\prime}}\right)\right) d \lambda\left(t^{\prime}\right) \\
& +\int_{0}^{t} \int_{0}^{t-t^{\prime}}\left(\bar{F}\left(\mathrm{x} e^{\delta\left(s^{\prime}+t^{\prime}\right)}\right)\right) d G\left(s^{\prime}\right) d \lambda\left(t^{\prime}\right)
\end{aligned}
$$

Holds uniformly $t \in \Lambda[0, T]$.

\section{Proof:}

$$
\begin{aligned}
& P\left(R_{\delta}^{*}(t, x)>x\right)=\left(\sum_{n=1}^{m_{0}}+\sum_{m_{0}+1}^{\infty}\right) \\
& P\left(\begin{array}{l}
X_{k} \mathrm{e}^{-\delta \omega_{\mathrm{k}}} 1_{\left\{\omega_{\mathrm{k}}<t\right\}}+Z_{k} \mathrm{e}^{-\delta\left(\omega_{\mathrm{k}}+T_{k}\right)} \\
1_{\left\{\omega_{\mathrm{k}}+T_{k}<t\right\}}>x, N(t)=n
\end{array}\right)=I_{1}+I_{2}
\end{aligned}
$$

Firstly, we deal with the $I_{1}$, according to the Lemma 4.1, it holds uniformly for $t \in \Lambda[0, T]$. We got:

$$
\begin{aligned}
& \mathrm{I}_{1} \sim\left(\sum_{n=1}^{m_{0}} \sum_{k=1}^{n}\right) P\left(X_{k} \mathrm{e}^{-\delta \omega_{\mathrm{k}}} 1_{\left\{\omega_{\mathrm{k}}<t\right\}}>\mathrm{x}, N(\mathrm{t})=\mathrm{n}\right) \\
& +\left(\sum_{n=1}^{m_{0}} \sum_{k=1}^{n}\right) P\left(Z_{k} \mathrm{e}^{-\delta\left(\omega_{\mathrm{k}}+T_{k}\right)} 1_{\left\{\omega_{\mathrm{k}}+T_{k}<t\right\}}>\mathrm{x}, N(\mathrm{t})=\mathrm{n}\right) \\
& =\left(\sum_{n=1}^{m_{0}} \sum_{n=m_{0}+1}^{n}\right) P\left(X_{k} \mathrm{e}^{-\delta \omega_{\mathrm{k}}} 1_{\left\{\omega_{\mathrm{k}}<t\right\}}>\mathrm{x}, N(\mathrm{t})=\mathrm{n}\right) \\
& \left.\left.+P\left(Z_{k} \mathrm{e}^{-\delta\left(\omega_{\mathrm{k}}+T_{k}\right)} 1_{\left\{\omega_{\mathrm{k}}+T_{k}<t\right\}}>>\mathrm{x}, N(\mathrm{t})=\mathrm{n}\right)\right)\right)=I_{3}-I_{4}
\end{aligned}
$$

By using the Lemma 3.1 and the independent relationship between $X_{k}, Z_{k}$ and $\omega_{k}$, we can exchange the $\sum_{n=1}^{m_{0}} \sum_{k=1}^{n}$ and $\sum_{k=1}^{n} \sum_{n=1}^{m_{0}}$, we can get:

$$
\begin{aligned}
& \mathrm{I}_{3}=\sum_{k=1}^{\infty} P\left(X_{k} \mathrm{e}^{-\delta \omega_{\mathrm{k}}} 1_{\left\{\omega_{\mathrm{k}}<t\right\}}>\mathrm{x}, N(\mathrm{t})=\mathrm{n}\right) \\
& +P\left(Z_{k} \mathrm{e}^{-\delta\left(\omega_{\mathrm{k}}+T_{k}\right)} 1_{\left\{\omega_{\mathrm{k}}+T_{k}<t\right\}}>\mathrm{x}, N(\mathrm{t})=\mathrm{n}\right) \\
& =\int_{0}^{t}\left(\bar{H}\left(\mathrm{x} e^{\delta t^{\prime}}\right)\right) d \lambda\left(t^{\prime}\right) \\
& +\int_{0}^{t} \int_{0}^{t-t^{\prime}}\left(\bar{F}\left(\mathrm{x}^{\delta\left(s^{\prime}+t^{\prime}\right)}\right)\right) d G\left(s^{\prime}\right) d \lambda\left(t^{\prime}\right)
\end{aligned}
$$

And then for $I_{4}$, because the independent relationship among $X_{k}, Z_{k}$ and $\omega_{k}$ and $H, F \in \mathcal{D}$ :

$$
\begin{gathered}
I_{4} \sum_{n=m_{0}+1}^{\infty} \sum_{k=1}^{n} P\left(X_{k} \mathrm{e}^{-\delta \omega_{\mathrm{k}}} 1_{\left\{\omega_{\mathrm{k}}<t\right\}}>x, N(\mathrm{t})=\mathrm{n}\right) \\
\left.+P\left(Z_{k} \mathrm{e}^{-\delta\left(\omega_{\mathrm{k}}+T_{k}\right)} 1_{\left\{\omega_{\mathrm{k}}+T_{k}<t\right\}}>\mathrm{x}, N(\mathrm{t})=\mathrm{n}\right)\right) \\
=\sum_{n=m_{0}+1}^{\infty}\left(\sum_{k=1}^{n} P\left(X_{k} \mathrm{e}^{-\delta \omega_{\mathrm{k}}} 1_{\left\{\omega_{\mathrm{k}}<t\right\}}>x\right)\right. \\
+\sum_{k=1}^{n} P\left(Z_{k} \mathrm{e}^{-\delta\left(\omega_{\mathrm{k}}+T_{k}\right)} 1_{\left\{\omega_{\mathrm{k}}+T_{k}<t\right\}}>\mathrm{x}\right) P(N(\mathrm{t})=\mathrm{n})
\end{gathered}
$$




$$
\begin{aligned}
& \leq \sum_{n=m_{0}+1}^{\infty} \mathrm{n}\left(P\left(X_{k}>x\right)+P\left(Z_{k}>x\right)\right) P(N(\mathrm{t})=\mathrm{n}) \\
& =\bar{H}(\mathrm{x}) n \sum_{n=m_{0}+1} P(N(\mathrm{t})=\mathrm{n})+\bar{F}(\mathrm{x}) \\
& n \sum_{n=m_{0}+1}^{\infty} P(N(\mathrm{t})=\mathrm{n}) \\
& =\bar{H}(\mathrm{x}) E N(t) 1_{\left\{\mathrm{N}(\mathrm{t})>\mathrm{m}_{0}\right\}}+\bar{F}(\mathrm{x}) E N(t) 1_{\left\{\mathrm{N}(\mathrm{t})>\mathrm{m}_{0}\right\}}
\end{aligned}
$$

By the condition $H \in \mathcal{D}$ and Lemma 3.3, we derive:

$$
\begin{aligned}
\lim _{\mathrm{m}_{0} \rightarrow \infty} \sup _{\mathrm{x} \rightarrow \infty} \sup _{\mathrm{t} \in \Lambda[0, \mathrm{~T}]} \frac{\mathrm{I}_{4}}{\int_{0}^{t}\left(\bar{H}\left(\mathrm{x} e^{\delta t^{\prime}}\right)\right) d \lambda\left(\mathrm{t}^{\prime}\right)}=0 \\
+\int_{0}^{t} \int_{0}^{t-t^{\prime}}\left(\bar{F}\left(\mathrm{x} e^{\delta\left(s^{\prime}+t^{\prime}\right)}\right)\right) \\
d G\left(s^{\prime}\right) d \lambda\left(\mathrm{t}^{\prime}\right)
\end{aligned}
$$

Before we turn to the $I_{2}$, we should introduce some basic probability theory related to $I_{2}$.

Because of the fact that $P(x+y>t) \leq P(x>\mathrm{t} / 2)+$ $P(y>t / 2)$, we get:

$$
\begin{aligned}
& P\left(\sum_{k=1}^{n} X_{k}+\sum_{k=1}^{n} Z_{k}>x\right) \leq P\left(\sum_{k=1}^{n} X_{k}>x / 2\right) \\
& +P\left(\sum_{k=1}^{n} Z_{k}>x / 2\right)
\end{aligned}
$$

Secondly, the basic rule in probability is that any event of probability should no greater than 1 :

$$
P\left(\sum_{k=1}^{n} X_{k}+\sum_{k=1}^{n} Z_{k}>x\right) \leq 1
$$

Thus:

$$
\begin{aligned}
& I_{2} \leq\left(\sum_{m_{0}<n \leq x / d}+\sum_{x / d<n \leq \infty}\right) \\
& P\left(\sum_{k=1}^{n} X_{k}+\sum_{k=1}^{n} Z_{k}>x\right) P(N(t)=n) \\
& \leq \sum_{\mathrm{m}_{0}<n \leq \mathrm{x} / \mathrm{d}}\left(P\left(\sum_{k=1}^{n} X_{k}>x / 2\right)\right. \\
& \left.+P\left(\sum_{k=1}^{n} Z_{k}>x / 2\right)\right) P(N(\mathrm{t})=\mathrm{n})+\sum_{x / d<n \leq \infty} P(N(\mathrm{t})=\mathrm{n})
\end{aligned}
$$

Applying the Markov's inequality and make corresponding substitution, we derive:

$$
P(|x|>a) \leq \frac{E(|x|)}{a} \leq \frac{E(|N(t)|)}{x / d}
$$

And combining the relation (2.6), we obtain:

$$
\begin{aligned}
& \frac{\bar{F}(\mathrm{x} / 2 \mathrm{n})}{\bar{F}(\mathrm{x})} \leq c(2 n)^{v} \leq c(n)^{v} \\
& \text { and } \frac{\bar{H}(\mathrm{x} / 2 \mathrm{n})}{\bar{H}(\mathrm{x})} \leq c(2 n)^{v} \leq c(n)^{v}
\end{aligned}
$$

We may also find two constants $\mathrm{c}$ and $\mathrm{d}$ such that for $\gamma>J_{H}^{+}$and $\gamma>J_{F}^{+}$under the condition of $H \in D$ and relation (2.6), $m_{0}+1 \leq x / d$, it holds uniformly for $t \in \Lambda[0, T]$. Therefore, from original inequality $I_{2}$ should yield:

$$
\begin{aligned}
& \quad I_{2} \leq \sum_{\mathrm{m}_{0}<n \leq \mathrm{x} / \mathrm{d}} \mathrm{n} \bar{H}(\mathrm{x} / 2 \mathrm{n}) P(N(\mathrm{t})=\mathrm{n}) \\
& \quad+\sum_{\mathrm{m}_{0}<n \leq \mathrm{x} / \mathrm{d}} \bar{F}(\mathrm{x} / 2 \mathrm{n}) P(N(\mathrm{t})=\mathrm{n})+P(N(\mathrm{t})>x / d) \\
& \leq c \bar{H}(x) \sum_{m_{0}<n \leq x / d} n^{\gamma+1} P(N(t)=n)+c \bar{F}(\mathrm{x}) \\
& \sum_{m_{0}<n \leq x / d} \mathrm{n}^{\gamma+1} P(N(\mathrm{t})=\mathrm{n}) \\
& +\left(\frac{\mathrm{x}}{\mathrm{d}}\right)^{\gamma+1} \mathrm{E}(\mathrm{N}(\mathrm{t}))^{\gamma+1} 1_{\{\mathrm{N}(\mathrm{t})>x / d\}} \\
& \leq c \bar{H}(\mathrm{x}) \mathrm{E}(\mathrm{N}(\mathrm{t}))^{\gamma+1} 1_{\left\{\mathrm{N}(\mathrm{t})>\mathrm{m}_{0}\right\}}+c \bar{F}(\mathrm{x}) \mathrm{E}(\mathrm{N}(\mathrm{t}))^{\gamma+1} 1_{\left\{\mathrm{N}(\mathrm{t})>\mathrm{m}_{0}\right\}}
\end{aligned}
$$

Hence, by the guaranteed condition of $H$ and $F$ belonging to the class $D$ and applying Lemma 3.3 to the following inequality again, we obtain:

$$
\begin{aligned}
& \lim _{m_{0} \rightarrow \infty} \operatorname{im}_{x \rightarrow \infty} \sup _{t \in \Lambda[0, T]} \frac{I_{2}}{\int_{0}^{t}\left(\bar{H}\left(\mathrm{x} e^{\delta t^{\prime}}\right)\right) d \lambda\left(\mathrm{t}^{\prime}\right)} \\
& +\int_{0}^{t} \int_{0}^{t-t^{\prime}}\left(\bar{F}\left(\mathrm{x} e^{\delta\left(s^{\prime}+t^{\prime}\right)}\right)\right) \\
& d G\left(s^{\prime}\right) d \lambda\left(\mathrm{t}^{\prime}\right) \\
& c \bar{H}(\mathrm{x}) \mathrm{E}(\mathrm{N}(\mathrm{t}))^{\gamma+1} 1_{\left\{\mathrm{N}(\mathrm{t})>\mathrm{m}_{0}\right\}} \\
& \leq \lim _{m_{0} \rightarrow \infty} \operatorname{im}_{x \rightarrow \infty} \sup _{t \in \Lambda[0, T]} \frac{+c \bar{F}(\mathrm{x}) \mathrm{E}(\mathrm{N}(\mathrm{t}))^{\gamma+1} 1_{\left\{\mathrm{N}(\mathrm{t})>\mathrm{m}_{0}\right\}}}{\bar{H}\left(\mathrm{x} e^{\delta T}\right) \lambda(\mathrm{t})+\bar{F}\left(\mathrm{x} e^{\delta T}\right) \lambda(\mathrm{t})} \\
& \leq c \lim _{x \rightarrow \infty} \frac{\bar{H}(\mathrm{x})+\bar{F}(\mathrm{x})}{\bar{H}\left(\mathrm{x} e^{\delta T}\right)+\bar{F}\left(\mathrm{x} e^{\delta T}\right)} \lim _{m_{0} \rightarrow \infty} \sup _{t \in \Lambda[0, T]} \\
& \lambda(\mathrm{t})^{-1} \mathrm{E}(\mathrm{N}(\mathrm{t}))^{\gamma+1} 1_{\left\{\mathrm{N}(\mathrm{t})>\mathrm{m}_{0}\right\}}=0
\end{aligned}
$$



(4.2).

Consequently, we complete the proof of Lemma

\section{Proof of Theorem 3.8}

Followed by the $\mathrm{Li}$ (2013) approach, we found that:

$$
\begin{aligned}
& \tilde{R}_{\delta}(t, x)=e^{(-\delta t)} R_{\delta}(t, x)=x \\
& +\int_{0}^{t} e^{-\delta s} c d s-\leq \sum_{k=1}^{\infty} X_{k} e^{-\delta \omega_{k}} 1_{\left\{\omega_{k} \leq t\right\}} \\
& -\sum_{k=1}^{\infty} Z_{k} e^{-\delta\left(\omega_{k}+T_{k}\right)} 1_{\left\{\omega_{k}+T_{k} \leq t\right\}}
\end{aligned}
$$

Then we rewrite it as:

$$
\tilde{R}_{\delta}(t, \mathrm{x})=\mathrm{x}+\tilde{c}(t)-R_{\delta}^{*}(t, x)
$$

Considering the ruin probability (1.5) in finite time in Risk Model section:

$$
\Phi(x, t)=P\left(R_{\delta}(x, \mathrm{~s})<0, \text { for some } 0 \leq \mathrm{s} \leq \mathrm{t}\right)
$$

Therefore:

$$
\Phi(x, t)=P\left(e^{-\delta t} R_{\delta}(x, \mathrm{~s})<0, \text { for some } 0 \leq \mathrm{s} \leq \mathrm{t}\right)
$$

It follows that:

$$
P\left(R_{\delta}^{*}(t, \mathrm{x})<x+\tilde{c}(t)\right) \leq \Phi(x, t) \leq P\left(R_{\delta}^{*}(t, \mathrm{x})>x\right)
$$

From which we can see that:

$$
x-R_{\delta}^{*}(t, \mathrm{x}) \leq \tilde{R}_{\delta}(t, \mathrm{x}) \leq \mathrm{x}+\frac{c}{\delta}\left(e^{\delta t}-1\right)-R_{\delta}^{*}(t, x)
$$

It follows that:

$$
P\left(R_{\delta}^{*}(t, \mathrm{x})>x+\frac{c}{\delta}\left(e^{\delta t}-1\right)\right) \leq \Phi(x, t) \leq P\left(R_{\delta}^{*}(t, \mathrm{x})>x\right)
$$

We have already proven the relation (4.11), so we can rewrite it:

$$
\begin{aligned}
& P\left(R_{\delta}^{*}(t, \mathrm{x})>x+\frac{c}{\delta}\left(e^{\delta t}-1\right)\right) \sim \int_{0}^{t}\left(\bar{H}\left(\mathrm{x}+\frac{\mathrm{c}}{\delta}\left(e^{\delta t}-1\right)\right) e^{\delta t^{\prime}}\right) d \lambda\left(t^{\prime}\right) \\
& +\int_{0}^{t} \int_{0}^{t-t^{\prime}}\left(\bar{F}\left(x+\frac{\mathrm{c}}{\delta}\left(e^{\delta t}-1\right)\right) e^{\delta\left(s^{\prime}+t^{\prime}\right)}\right) d G\left(s^{\prime}\right) d \lambda\left(t^{\prime}\right)
\end{aligned}
$$

Then for any $\varepsilon>0$, due to the condition (2.6) in preliminary section, we find that:

$$
\begin{aligned}
& P\left(R_{\delta}^{*}(t, \mathrm{x})>x+\frac{c}{\delta}\left(e^{\delta t}-1\right)\right) \geq \int_{0}^{t}\left(\bar{H}(1+\varepsilon) x e^{\delta t^{\prime}}\right) d \lambda\left(t^{\prime}\right) \\
& +\int_{0}^{t} \int_{0}^{t-t^{\prime}}\left(\bar{F}(1+\varepsilon) x e^{\delta\left(s^{\prime}+t^{\prime}\right)}\right) d G\left(s^{\prime}\right) d \lambda\left(t^{\prime}\right) \\
& \geq(1+\varepsilon)^{\eta}\left(\begin{array}{l}
\int_{0}^{t}\left(\bar{H}\left(x e^{\delta t^{\prime}}\right)\right) d \lambda\left(t^{\prime}\right) \\
+\int_{0}^{t} \int_{0}^{t-t^{\prime}}\left(\bar{F}\left(\mathrm{x} e^{\delta t^{\prime}}\right)\right) d G\left(s^{\prime}\right) d \lambda\left(t^{\prime}\right)
\end{array}\right)
\end{aligned}
$$

By the arbitrariness of $\varepsilon>0$, it follows that:

$$
\begin{aligned}
& P\left(R_{\delta}^{*}(t, \mathrm{x})>x+\frac{c}{\delta}\left(e^{\delta t}-1\right)\right) \geq \int_{0}^{t}\left(\bar{H}\left(x e^{\delta t^{\prime}}\right)\right) d \lambda\left(t^{\prime}\right) \\
& +\int_{0}^{t} \int_{0}^{t-t^{\prime}}\left(\bar{F}\left(x e^{\delta t^{\prime}}\right)\right) d G\left(s^{\prime}\right) d \lambda\left(t^{\prime}\right)
\end{aligned}
$$

And then, similarly, we can also obtain the remaining part:

$$
\begin{aligned}
& P\left(R_{\delta}^{*}(t, \mathrm{x})>x\right) \sim \int_{0}^{t}\left(\bar{H}\left(x e^{\delta y}\right)\right) d y \\
& +\int_{0}^{t} \int_{0}^{t-t^{\prime}}\left(\bar{F}\left(x e^{\delta t^{\prime}}\right)\right) d G\left(s^{\prime}\right) d y
\end{aligned}
$$

This completes the proof of Theorem (3.8).

\section{CONCLUSION}

Enlightened by the results of $(\mathrm{Li}, 2013$; Wang et al., 2013), we obtained some novel results regarding the pSQAI and WLOD random variables within the class $\mathcal{L} \cap \mathcal{D}$. Our main results concerned the approximation for constant interest rate and by-claim model. In addition, we further prove the main results and corresponding assumptions. The asymptotic behavior of $P\left(R_{\delta}^{*}(t, x)>x\right)$ is a key role in our proof part. Finally, we apply the obtained results to a kind of claim-dependent risk model and derive a more precise and more general asymptotic formula for ruin probability in finite time.

\section{ACKNOWLEDGEMENT}

The researcher would like to thank some comments and constructive suggestions of my advisor and all the 
members of committee. Several stimulating discussions allowed me to develop original ideas and improve my paper.

\section{REFERENCE}

Chen, Y. and K.W. Ng, 2007. The ruin probability of the renewal model with constant interest force and negatively dependent heavy-tailed claims. Insur. Math. Econ., 40: 415-423. DOI: 10.1016/j.insmatheco.2006.06.004

Embrechts, P., C. Kluppelberg and T. Mikosch, 1997. Modelling Extremal Events: For Insurance and Finance. 1st Edn., Springer Science and Business Media, Berlin, ISBN-10: 3540609318, pp: 645.

Hao, X. and Q. Tang, 2008. A uniform asymptotic estimate for discounted aggregate claims with subexponential tails. Insur. Math. Econ., 43: 116120. DOI: 10.1016/j.insmatheco.2008.03.009

Kluppelberg, C. and U. Stadtmuller, 1998. Ruin probabilities in the presence of heavy-tails and interest rates. Scand. Actuar. J., 1998: 49-58. DOI: 10.1080/03461238.1998.10413991

Lehmann, E.L., 1966. Some concepts of dependence. Annals Math. Stat., 37: 1137-1153.

Li, J., K. Wang and Y. Wang, 2009. Finite-time ruin probability with NQD dominated varying-tailed claims and NLOD inter-arrival times. J. Syst. Sci. Complex., 22: 407-414. DOI: 10.1007/s11424009-9173-7

Li, J., 2013. On pairwise quasi-asymptotically independent random variables and their applications. Stat. Probab. Lett., 83: 2081-2087. DOI: $10.1016 /$ j.spl.2013.05.023

Liu, X., Q. Gao and Y. Wang, 2012. A note on a dependent risk model with constant interest rate. Stat. Probab. Lett., 82: 707-712. DOI: 10.1016/j.spl.2011.12.016

Lundberg, F., 1903. Configuration approximated front of the probability function. II. FBK orsakringav collective risks, Almqvist and Wiksell, Uppsala.

Shen, X. and Z. Lin, 2008. Precise large deviations for randomly weighted sums of negatively dependent random variables with consistently varying tails. Stat. Probab. Lett., 78: 3222-3229. DOI: 10.1016/j.spl.2008.06.007
Tang, Q. and G. Tsitsiashvili, 2003. Randomly weighted sums of subexponential random variables with application to ruin theory. Extremes, 6: 171-188. DOI: 10.1023/B:EXTR.0000031178.19509.57

Tang, Q., 2006. Insensitivity to negative dependent of the asymptotic behavior of precise large. Electron J. Probab., 11: 107-120.

Tang, Q., 2005. The finite time ruin probability of the compound Poisson model with constant interest force. J. Applied Pro., 42: 608-619.

Wang, D., 2008. Finite-time ruin probability with heavytailed claims and constant interest rate. Stoch. Models, 24: 41-57, 2008. DOI: $10.1080 / 15326340701826898$

Wang, K., Y. Wang and Q. Gao, 2013. Uniform asymptotics for the finite-time ruin probability of a dependent risk model with a constant interest rate. Methodol. Comput. Applied Probab., 15: 109-124. DOI: $10.1007 / \mathrm{s} 11009-011-9226-\mathrm{y}$

Weng, C., Y. Zhang and S. Tan, 2012. Tail behavior of poisson shot noise processes under heavy-tailed shocks and actuarial applications. Methodol. Comput. Applied Probab., 15: 655-682. DOI: 10.1007/s11009-011-9274-3

Yang, Y. and Y. Wang, 2010. Asymptotics for ruin probability of some negatively dependent risk models with a constant interest rate and dominatedly-varying-tailed claims. Stat. Probab. Lett., 80: 143154. DOI: 10.1016/j.spl.2009.09.023

Yuen, K., J. Guo and K. Wang, 2005. On ultimate ruin in a delayed-claims risk model. J. Applied Prob., 42: 163-174. DOI: 10.1239/jap/1110381378

Waters, H.R. and A. Papatriandafylou, 1983. Ruin probabilities allowing for delay in claims settlement. Insur. Math. Econ., 4: 113-122. DOI: 10.1016/01676687(85)90005-8

Kabanov, Y.M., R.S. Liptser and A.N. Shiryaev, 1986. On the variation distance for probability measures defined on a filtered space. Probab. Theory Related Fields, 71: 19-35. DOI: 10.1007/BF00366270 